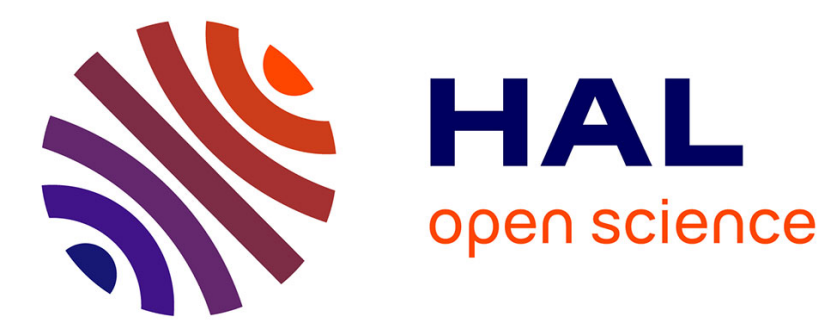

\title{
Uniform limit theorems for the integrated periodogram of weakly dependent time series and their applications to Whittle's estimate
}

\author{
Jean-Marc Bardet, Paul Doukhan, José Rafael León
}

\section{- To cite this version:}

Jean-Marc Bardet, Paul Doukhan, José Rafael León. Uniform limit theorems for the integrated periodogram of weakly dependent time series and their applications to Whittle's estimate. Journal of Time Series Analysis, 2008, 29 (5), pp.906-945. 10.1111/j.1467-9892.2008.00588.x . hal-00126489

\section{HAL Id: hal-00126489 \\ https://hal.science/hal-00126489}

Submitted on 25 Jan 2007

HAL is a multi-disciplinary open access archive for the deposit and dissemination of scientific research documents, whether they are published or not. The documents may come from teaching and research institutions in France or abroad, or from public or private research centers.
L'archive ouverte pluridisciplinaire HAL, est destinée au dépôt et à la diffusion de documents scientifiques de niveau recherche, publiés ou non, émanant des établissements d'enseignement et de recherche français ou étrangers, des laboratoires publics ou privés. 


\title{
Uniform limit theorems for the integrated periodogram of weakly dependent time series and their applications to Whittle's estimate
}

\author{
Jean-Marc Bardet ${ }^{a *}$ Paul Doukhan ${ }^{a b}$, José Rafael León ${ }^{c \dagger}$ \\ ${ }^{a}$ SAMOS-MATISSE-CES, Université Paris I, 90 rue de Tolbiac, 75013 Paris, FRANCE \\ bardet@univ-paris1.fr \\ ${ }^{b}$ LS-CREST, Timbre J340, 3 avenue Pierre Larousse, 92240 Malakoff, FRANCE \\ doukhan@ensae.fr \\ ${ }^{c}$ Universidad Central de Venezuela, Escuela de Matemática. \\ Ap. 47197 Los Chaguaramos, Caracas 1041-A VENEZUELA \\ jleon@euler.ciens.ucv.ve
}

\begin{abstract}
We prove uniform convergence results for the integrated periodogram of a weakly dependent time series, namely a law of large numbers and a central limit theorem. These results are applied to Whittle's parametric estimation. Under general weak-dependence assumptions we derive uniform limit theorems and asymptotic normality of Whittle's estimate for a large class of models. For instance the causal $\theta$-weak dependence property allows a new and unified proof of those results for $\operatorname{ARCH}(\infty)$ and bilinear processes. Non causal $\eta$-weak dependence yields the same limit theorems for two-sided linear (with dependent inputs) or Volterra processes.
\end{abstract}

Key words: Periodogram, Weak dependence, Whittle estimate.

AMS Subject Classification: 60F17, 60F25, 62M09, 62M10, 62M15.

Running title: Uniform limit theorems for the integrated periodogram and Whittle's estimate.

\section{Introduction}

Parametric estimation from a sample of a stationary time series is an important statistic problem both for theoretical research and for its practical applications to real data. Whittle's approximation likelihood estimate is particularly attractive for numerous models like ARMA, linear processes, etc. mainly for two reasons: first, Whittle's contrast does not depend on the marginal law of the time series but only on its spectral density, and second, its computation time is smaller than other parametric estimation methods such as exact likelihood. Numerous papers have been written on this estimation method after Whittle's seminal paper and in particular Hannan (1973), Rosenblatt (1985), and Giraitis and Robinson (2001), established, respectively, the asymptotic normality for Gaussian and causal linear, strong mixing and $\operatorname{ARCH}(\infty)$ processes. The case of long range dependent processes, which is not considered here, was studied by Fox and Taqqu (1986), and Giraitis and Surgailis (1990) (note also that in a semi-parametric frame, Robinson (1995) and recently Dalla et al. (2006) proposed an estimator of the long-memory parameter based on local Whittle estimation). The main goal of the present paper is to provide a unified treatment of this asymptotic normality for a very rich class of weakly dependent time processes, including those previously mentioned, but also some never studied non causal or non linear processes.

More precisely, let $X=\left(X_{k}\right)_{k \in \mathbb{Z}}$ be a zero mean fourth-order stationary time series with real values. Denote by $(R(s))_{s}$ the covariogram of $X$, and by $\left(\kappa_{4}(i, j, k)\right)_{i, j, k}$ the fourth cumulants of $X$ defined by

$$
\begin{aligned}
R(s) & =\operatorname{Cov}\left(X_{0}, X_{s}\right)=\mathbb{E}\left(X_{0} X_{s}\right), \quad \text { for } s \in \mathbb{Z}, \\
\kappa_{4}(i, j, k) & =\mathbb{E} X_{0} X_{i} X_{j} X_{k}-\mathbb{E} X_{0} X_{i} \mathbb{E} X_{j} X_{k}-\mathbb{E} X_{0} X_{j} \mathbb{E} X_{i} X_{k}-\mathbb{E} X_{0} X_{k} \mathbb{E} X_{i} X_{j}, \quad \text { for }(i, j, k) \in \mathbb{Z}^{3} .
\end{aligned}
$$

${ }^{*}$ Author for correspondence.

${ }^{\dagger}$ This author aknowledges the program ECOS-NORD of Fonacit, Venezuela, for its support. 
We will use the following assumption on $X$

Assumption M: $X$ is such that

$$
\gamma=\sum_{\ell \in \mathbb{Z}} R(\ell)^{2}<\infty \text { and } \kappa_{4}=\sum_{i, j, k}\left|\kappa_{4}(i, j, k)\right|<\infty
$$

The periodogram of $X$ is

$$
I_{n}(\lambda)=\frac{1}{2 \pi \cdot n}\left|\sum_{k=1}^{n} X_{k} e^{-i k \lambda}\right|^{2}, \quad \text { for } \lambda \in[-\pi, \pi[
$$

Now, let $g: \mathbb{R} \rightarrow \mathbb{R}$ a $2 \pi$-periodic function such that $g \in \mathbb{L}^{2}([-\pi, \pi[)$ and define

$$
\begin{aligned}
J_{n}(g) & =\int_{-\pi}^{\pi} g(\lambda) I_{n}(\lambda) d \lambda, \quad \text { the integrated periodogram of } X \\
\text { and } J(g) & =\int_{-\pi}^{\pi} g(\lambda) f(\lambda) d \lambda,
\end{aligned}
$$

with $f$ denoting the spectral density of $X$ (that exists and is in $\mathbb{L}^{2}([-\pi, \pi[)$ from Assumption M) defined by

$$
f(\lambda)=\frac{1}{2 \pi} \sum_{k \in \mathbb{Z}} R(k) e^{i k \lambda} \text { for } \lambda \in[-\pi, \pi[
$$

Recall that $I_{n}(\lambda)=\frac{1}{2 \pi} \sum_{|k|<n} \widehat{R}_{n}(k) e^{-i k \lambda}$ with $\widehat{R}_{n}(k)=\frac{1}{n} \sum_{j=1 \vee(1-k)}^{(n-k) \wedge n} X_{j} X_{j+k}$, which is a biased estimate of $R(k)$. Thus, the periodogram $I_{n}(\lambda)$ could be a natural estimator of the spectral density; unfortunately it is not a consistent estimator. However, once integrated with respect to some $\mathbb{L}^{2}$ function, its behavior becomes quite smoother and can allow an estimation of the spectral density. A special case of the integrated periodogram is Whittle's contrast, defined as a function $\beta \rightarrow J_{n}\left(h_{\beta}\right)$, where $h_{\beta}$ is included in a class of functions depending on the vector of parameters $\beta$. Whittle estimator minimizes this contrast. As a consequence, uniform limit theorems for the integrated periodogram $J_{n}(\cdot)$ are the appropriate tools for obtaining uniform limit theorems for Whittle's contrast, that imply, under additional conditions concerning the regularity of the spectral density, limit theorems for Whittle's estimators.

A uniform strong law of large numbers of integrated periodograms on a Sobolev-type space (included in the space of $2 \pi$-periodic $\mathbb{L}^{2}$-functions) is first established only under assumption $\mathrm{M}$. Additional assumptions on the dependence properties of the time series have to be specified for establishing central limit theorems. Our choice has been to consider time series satisfying weak dependence properties introduced and developed in Doukhan and Louhichi (1999). Numerous reasons may explain this choice. First, this frame of dependence includes a lot of models like causal or non causal linear, bilinear, strong mixing processes and also dynamical systems. Second, they are independent of the marginal distribution of the time series. Finally, they can be easily used in various statistical contexts, in particular in the case of the integrated periodogram which is a quadratic form. These uniform limit theorems can be compared with those obtained in Dahlhaus (1988) or Mikosch and Norvaisa (1997). Roughly speaking, the presented results are obtained under weaker conditions on time series, but considering different functional spaces.

Two frames of weak dependence are considered here. The first one exploits a causal property of dependence, the $\theta$-weak dependence property (see Dedecker and Doukhan, 2003). Under certain conditions, the uniform limit theorems for integrated periodogram and asymptotic normality of Whittle's estimate are established. These general results are new and extend Hannan's (1973) and Rosenblatt's (1985) classical results for causal linear or strong mixing processes. For example, parametric and causal ARCH $(\infty)$ or bilinear processes (a very general class of models introduced by Giraitis and Surgailis (2002), see definition (21)) are considered; under certain conditions, the asymptotic normality of Whittle's estimators for those two classes of models is established with the same method. (The case of causal $\operatorname{ARCH}(\infty)$, and therefore of $\operatorname{GARCH}(\mathrm{p}, \mathrm{q})$, was already treated by Giraitis and Robinson, 2001, under less restrictive conditions. However, their proof is ad hoc and cannot be used in a more general context).

The second type of dependence under consideration is $\eta$-weak dependence. This property allows to derive central limit theorems for non causal processes, see [2] or [19]. These results can be applied for instance, to two-sided linear or Volterra processes (see their definition below in Section 3). Let us remark that usual proofs 
of central limit theorems for the integrated periodogram are established by considering increments of martingales or asymptotic results for strong mixing processes, which is not a method adapted for non causal processes, even in the simple case of two-sided linear models. The proof of our results is a corollary of a general functional central limit theorem for $\eta$-weakly dependent processes, established by using Bernstein's blocks method. Even if our results may be sub optimal in terms of the conditions linking the moment assumption with the decay rate of weak dependence of the time series, they however cover numerous models and open new perspectives of treatment for non causal processes.

The paper is organized as follows. In Section 2, uniform limit theorems are presented with some applications to time series. Section 3 is devoted to limit theorems satisfied by Whittle's estimators, that are applied to several examples of causal and non causal processes. Section 1 contains the main proofs, and a useful lemma is presented in an appendix (Section 5).

\section{Uniform limit theorems}

\subsection{Notations and assumptions}

Afterwards we shall use the zero mean random variables

$$
Y_{j, k}=X_{j} X_{j+k}-R(k), \text { for all }(j, k) \in \mathbb{Z}^{2} .
$$

We intend to work in a Sobolev space $\mathcal{H}_{s}$ of locally $\mathbb{L}^{2}$ and $2 \pi$-periodic functions, defined by

$$
\mathcal{H}_{s}=\left\{g \in \mathbb { L } ^ { 2 } \left(\left[-\pi, \pi[) /\|g\|_{\mathcal{H}_{s}}<\infty\right\} \quad \text { with }\|g\|_{\mathcal{H}_{s}}^{2}=\sum_{\ell \in \mathbb{Z}}(1+|\ell|)^{2 s} \cdot\left|g_{\ell}\right|^{2}, \quad \text { and } s>1 / 2\right.\right.
$$

where $g(\lambda)=\sum_{\ell \in \mathbb{Z}} g_{\ell} e^{i \ell \lambda}$. This space $\mathcal{H}_{s}$ is included in the space $C^{\star}$ of continuous and $2 \pi-$ periodic functions and $\|g\|_{\infty}=\sup _{[-\pi, \pi[}|g| \leq \sqrt{c}_{s} \cdot\|g\|_{\mathcal{H}_{s}}$ with

$$
c_{s}=\sum_{\ell \in \mathbb{Z}}(1+|\ell|)^{-2 s}
$$

As usual $\mathcal{H}_{s}^{\prime}$ denotes the dual of $\mathcal{H}_{s}$ with norm defined from the identity $\|T\|_{\mathcal{H}_{s}^{\prime}}=\sup _{\|g\|_{\mathcal{H}_{s}} \leq 1}|T(g)|$. Hence if $T \in \mathcal{H}_{s}^{\prime}$

$$
\|T\|_{\mathcal{H}_{s}^{\prime}}^{2}=\sup _{\|g\|_{\mathcal{H}_{s} \leq 1}}|T(g)|^{2}=\sum_{\ell \in \mathbb{Z}}(1+|\ell|)^{-2 s} \cdot\left|T\left(e^{i \ell \lambda}\right)\right|^{2} .
$$

We study the asymptotic behavior of $J_{n}-J$ uniformly in the function space $\mathcal{H}_{s}$ or equivalently as elements of the Hilbert space $\mathcal{H}_{s}^{\prime}$.

\subsection{Uniform Strong Law of Large Numbers for the integrated periodogram}

We develop a uniform strong law of large numbers (Uniform SLLN) for the integrated periodogram $\left(J_{n}(g)\right)_{g}$. An important feature is that the assumptions are only stated here in terms of cumulant sums. Thus we need no additional assumption on the dependence of the sequence $X$.

Theorem 1 (ULLN) If $X$ satisfies Assumption $M$, then

$$
\left\|J_{n}-J\right\|_{\mathcal{H}_{s}^{\prime}} \underset{n \rightarrow \infty}{\stackrel{a . s .}{\longrightarrow}} 0 .
$$

Remark Let $X$ be a 4 -th order causal linear process, i.e. $X_{n}=\sum_{k=0}^{\infty} a_{k} \xi_{n-k}$ for $n \in \mathbb{Z}$, where $\left(\xi_{k}\right)_{k \in \mathbb{Z}}$ is a sequence of zero mean i.i.d.r.v. such that $\sum_{k} k a_{k}^{2}<\infty, \sum_{k}\left|a_{k}\right|<\infty$ and $\mathbb{E} \xi_{0}^{4}<\infty$. From Rosenblatt (1985, p. 59), and the multilinearity of cumulants (i.e. $\left|\kappa_{4}\right| \leq \sum_{i, j, k}\left|\kappa_{4}(i, j, k)\right|=\left|c_{4}\right|\left(\sum_{k}\left|a_{k}\right|\right)^{4}$ where $c_{4}$ is the 4 -th order cumulant of $\left(\xi_{k}\right)_{k \in \mathbb{Z}}$ ), then $X$ satisfies Assumption M and therefore the previous uniform SLLN yields. In Mikosch and Norvaisa (1997), uniform LLN for the integrated periodogram are also proved for causal linear processes. The integrated periodogram is then considered on a general class of functions $\mathcal{F}$ endowed with a pseudometric space of $\mathcal{L}^{2}$. In addition of the fourth moment condition, the assumptions are respectively in Theorems 3.3 and 3.4, $\sum_{k=0}^{\infty} k a_{k}^{2}<\infty$ for a uniform weak LLN and $\sum_{j=1}^{\infty} k^{3 / 2} a_{k}^{2}<\infty$ for a uniform strong LLN. As a conclusion, the uniform strong LLN (3) is satisfied by a larger class of causal linear processes but the functional space $\mathcal{H}_{s}$ is different from $\mathcal{F}$ (with also a different distance). 


\subsection{Uniform Central Limit Theorem for the integrated periodogram}

Now, we would like to establish a uniform central limit theorem (UCLT) for the integrated periodogram $J_{n}(\cdot)$ on the space of functions $\mathcal{H}_{s}$. Assumption $\mathrm{M}$ is not sufficient for such a result. The dependence between the terms of the time series $X$ has to be specified, and we will consider 2 cases. Before this, under Assumption M, we define for any $\lambda, \mu, \nu \in \mathbb{R}$, the bispectral density

$$
f_{4}(\lambda, \mu, \nu)=\frac{1}{(2 \pi)^{3}} \sum_{h=-\infty}^{\infty} \sum_{k=-\infty}^{\infty} \sum_{\ell=-\infty}^{\infty} \kappa_{4}(h, k, \ell) e^{i(h \lambda+k \mu+\ell \nu)}
$$

the matrix $\Sigma=\left(\sigma_{\ell_{i}, \ell_{j}}\right)_{1 \leq i, j \leq m}$, where $\ell_{i}$ are distinct integer numbers, with

$$
\sigma_{k, \ell}=\sum_{h \in \mathbb{Z}}\left(R(h) R(h+\ell-k)+R(h+\ell) R(h-k)+\kappa_{4}(h, k, h+\ell)\right),
$$

and for $g_{1}$ and $g_{2}$ in $\mathcal{H}_{s}$, the limiting covariance $\Gamma\left(g_{1}, g_{2}\right)$

$$
\Gamma\left(g_{1}, g_{2}\right)=4 \pi \int_{-\pi}^{\pi} g_{1}(\lambda) g_{2}(\lambda) f^{2}(\lambda) d \lambda+2 \pi \int_{-\pi}^{\pi} \int_{-\pi}^{\pi} g_{1}(\lambda) g_{2}(\mu) f_{4}(\lambda,-\mu, \mu) d \lambda d \mu .
$$

\subsubsection{UCLT for causal time series}

This first case follows a classical methodology: the UCLT results from the finite dimensional convergence and the tightness of the process $Z=\left(Z_{n}(g)\right)_{g \in \mathcal{H}_{s}}$ where $Z_{n}(g)=\sqrt{n}\left(J_{n}(g)-J(g)\right)$ for $n \in \mathbb{N}^{*}$ and $g \in \mathcal{H}_{s}$. Since $g \mapsto Z_{n}(g)$ is a linear functional, the finite dimensional convergence is a consequence of the multidimensional central limit theorem for empirical covariances.

In the sequel, for $\ell \in \mathbb{Z}$, we will denote by $\mathcal{M}_{0}^{(\ell)}$ a $\sigma$-algebra such that

$$
\mathcal{M}_{0}^{(\ell)} \supset \sigma\left(Y_{k, \ell}, k \leq 0\right)=\sigma\left(X_{k} X_{k+\ell}, k \leq 0\right) .
$$

Here $\sigma\left(W_{i}, \quad i \in I\right)$ represents the $\sigma$-algebra generated by $\left(W_{i}\right)_{i \in I}$. An example of such $\sigma$-algebra $\mathcal{M}_{0}^{(\ell)}$ is $\mathcal{M}_{0}^{(\ell)}=\sigma\left(X_{k_{0}}, X_{k_{0}+\ell}\right)$ for a given $k_{0} \in \mathbb{Z}$. The most classical of such $\sigma$-algebra is defined, for any integer $p$ such that $p \geq \ell$,

$$
\mathcal{M}_{0}^{(\ell)}=\mathcal{B}_{p}=\sigma\left(X_{j}, j \leq p\right)
$$

Lemma 1 Let $\left(\ell_{1}, \ldots, \ell_{m}\right) \in \mathbb{Z}^{m}$ be arbitrary distinct non-negative integers $\left(m \in \mathbb{N}^{*}\right)$. Let $X$ satisfy Assumption $M$ and be such that

$$
\sum_{k \geq 0}\left|\mathbb{E}\left(Y_{0, \ell_{i}} \mathbb{E}\left(Y_{k, \ell_{i}} \mid \mathcal{M}_{0}^{\left(\ell_{i}\right)}\right)\right)\right|<\infty \quad \text { for all } i \in\{1, \ldots, m\} .
$$

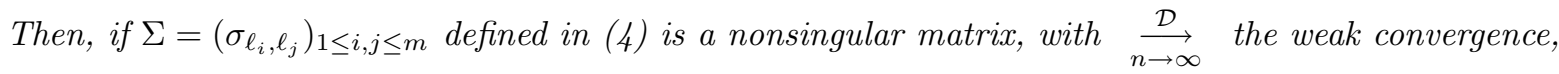

$$
\left(\sqrt{n}\left(\widehat{R}_{n}\left(\ell_{i}\right)-\mathbb{E} \widehat{R}_{n}\left(\ell_{i}\right)\right)\right)_{1 \leq i \leq m} \underset{n \rightarrow \infty}{\stackrel{\mathcal{D}}{\longrightarrow}} \mathcal{N}_{m}(0, \Sigma)
$$

Remark Lemma 1 is a generalization of a result of Rosenblatt (1985, Theorem 3, p. 58) which asserts the CLT if for each $i \in\{1, \ldots, m\}, \sum_{k=0}^{\infty}\left(\mathbb{E}\left(\mathbb{E}\left(Y_{k, \ell_{i}} \mid \mathcal{B}_{0}\right)\right)^{2}\right)^{1 / 2}<\infty$, and this implies (7). An analogue CLT in Hall and Heyde (1980) (Theorem 5.4, page 136) does not seem to be adapted to work out the forthcoming examples.

Theorem 2 Under assumptions of Lemma 1, the Uniform Central Limit Theorem (UCLT) holds

$$
Z_{n}=\sqrt{n}\left(J_{n}-J\right) \underset{n \rightarrow \infty}{\stackrel{\mathcal{D}}{\longrightarrow}} Z \text { in the space } \mathcal{H}_{s}^{\prime},
$$

with $(Z(g))_{g \in \mathcal{H}_{s}}$ the zero mean Gaussian process with covariance $\Gamma\left(g_{1}, g_{2}\right)$ defined in (5). 
Remark Dahlhaus (1988) and Mikosch and Norvaisa (1997) established also UCLT of the integrated periodogram for a general class of multivariate processes with high-order spectra (including certain Gaussian processes) and for causal linear processes, respectively. The considered functional spaces require to satisfy conditions close to the classical Dudley's conditions. The UCLT theorem 2 needs the simple assumption $s>\frac{1}{2}$, besides the conditions for finite dimensional convergence. Recall that assumption $s>\frac{1}{2}$ is also required for obtaining uniform equicontinuity, however additional dependence conditions are always needed (see, for example, Dedecker and Louhichi, 2002). The book edited by Dehling et al. (2002) contains also two other articles on empirical spectral processes, concerned respectively with uniform results for Gaussian processes (Dahlhaus and Polonik, 2002) and long range dependence processes (Soulier, 2002). Tails of the variables and measures of entropy need to be controlled as this is usual for empirical processes (see the paper by Dedecker and Louhichi in the same monograph); we do not have such assumption to the price of very specific Hilbert type classes. An important feature is the fact that assumption $s>\frac{1}{2}$ is the best possible assumption for empirical CLT in order that the corresponding Dudley entropy integral converges. Uniform theorems on Hilbert classes are indeed usually weaker results as stated in several works of Suquet (see for instance Oliveira and Suquet, 1998); using De Acosta (1970)'s criterion, entails that essentially the same assumptions that those ensuring fidi convergence usually implies functional uniform convergence in Hilbert spaces.

Examples of time series satisfying Theorem 2. This theorem is first applied to 3 classical examples of time series, which extend the known multidimensional CLT for integrated periodogram. Then, the UCLT is established for a very rich class of causal time series, the $\theta$-weakly dependent processes.

1. Causal linear processes: let $X$ be a linear and causal time series such that $X_{n}=\sum_{k=0}^{\infty} a_{k} \xi_{n-k}$ for $n \in \mathbb{Z}$, where $\left(\xi_{k}\right)_{k \in \mathbb{Z}}$ is a sequence of zero mean independent identically distributed random variables such that $\mathbb{E} \xi_{0}^{4}<\infty$. Assume also that the real sequence $\left(a_{k}\right)$ satisfied $\sum_{k} k a_{k}^{2}<\infty$ and $\sum_{k}\left|a_{k}\right|<\infty$ (these conditions are weaker than those of Mikosch and Norvaisa, 1997, which are $\sum_{k} k^{3 / 2+\varepsilon} a_{k}^{2}<\infty$ with $\varepsilon>0$, but the considered function spaces in both the UCLT are different). Thus $X$ satisfies assumption $\mathrm{M}$ and from Rosenblatt (1985, p. 59), we have for all $\ell \in \mathbb{N}, \sum_{k=0}^{\infty}\left\|\mathbb{E}\left(Y_{k, \ell} \mid \mathcal{B}_{\ell}\right)\right\|_{2}<\infty$ and thus (7) is also satisfied. Then the UCLT (9) holds.

2. Gaussian processes: let the sequence $\left(X_{n}\right)_{n \in \mathbb{Z}}$ be a zero mean stationary Gaussian process such that $\sum_{k} R(k)^{2}<\infty$. Then $X$ satisfies Assumption $\mathrm{M}$ for all $\ell \in \mathbb{Z}$ and $k \in \mathbb{N}$, and, with the $\sigma$-algebra defined by (6) for $m=k+\ell,\left|\mathbb{E}\left(Y_{0, \ell} \mathbb{E}\left(Y_{k, \ell} \mid \mathcal{M}_{0}^{(\ell)}\right)\right)\right|=\left|\mathbb{E}\left(X_{0} X_{\ell} X_{k} X_{k+\ell}\right)-R(\ell)^{2}\right|$. But

$$
\mathbb{E}\left(X_{0} X_{\ell} X_{k} X_{k+\ell}\right)-R(\ell)^{2}=R(k)^{2}+R(k+\ell) R(k-\ell)
$$

for a zero mean stationary Gaussian process. Thus,

$$
\sum_{k \geq 0}\left|\mathbb{E}\left(Y_{0, \ell} \mathbb{E}\left(Y_{k, \ell} \mid \mathcal{M}_{0}^{(\ell)}\right)\right)\right| \leq \sum_{k \in \mathbb{Z}} R(k)^{2}+\left(\sum_{k \in \mathbb{Z}} R^{2}(k+\ell)\right)^{1 / 2} \cdot\left(\sum_{k \in \mathbb{Z}} R^{2}(k-\ell)\right)^{1 / 2},
$$

from the Cauchy-Schwarz inequality for $\ell^{2}$ sequences. Therefore the UCLT (9) applies and the assumption $\left(\sum_{k} R(k)^{2}<\infty\right)$ seems to be sharp in this case.

3. Strong mixing processes: here, we consider the probability space $(\Omega, \mathcal{T}, \mathbb{P})$.

Corollary 1 Let $X=\left(X_{n}\right)_{n \in \mathbb{Z}}$ be a sequence of random variables on $(\Omega, \mathcal{T}, \mathbb{P})$ satisfying Assumption $M$. Assume that $X$ is a $\alpha^{\prime}$-mixing process, i.e.

$$
\alpha_{n}^{\prime}=\sup _{\ell \geq 0}\left\{\alpha\left(\sigma\left(X_{n}, X_{n+\ell}\right), \mathcal{B}_{0}\right)\right\} \underset{n \rightarrow \infty}{\longrightarrow} 0,
$$

where $\alpha(\mathcal{A}, \mathcal{B})=\sup _{A \in \mathcal{A}, B \in \mathcal{B}}|\mathbb{P}(A \cap B)-\mathbb{P}(A) \mathbb{P}(B)|$ for $\mathcal{A}, \mathcal{B} \subset \mathcal{T}$. Moreover, with $Q_{|X|}$ denoting the quantile function of $|X|$ and $\alpha^{\prime-1}(u)=\sum_{k \geq 0} \mathbb{1}_{u \leq \alpha_{k}^{\prime}}$, assume that $\int_{0}^{1} \alpha^{\prime-1}(u) Q_{\left|X_{0}\right|}^{4}(u) d u<\infty$. Then the UCLT (9) holds. 
Remark We note that $\alpha_{n}^{\prime} \leq \alpha_{n}=\alpha\left(\sigma\left(X_{k}, k \geq n\right), \mathcal{B}_{0}\right)$ the standard mixing coefficient in Rosenblatt (1985). However, no simple counter example seems to be available. Therefore, if $X$ is a strongly $\alpha$-mixing process satisfying Assumption M such that $\int_{0}^{1} \alpha^{-1}(u) Q_{\left|X_{0}\right|}^{4}(u) d u<\infty$, the UCLT (9) also holds.

4. Causal $\theta$-weakly dependent processes: Doukhan and Louhichi (1999) introduced the class of $\theta$-weakly dependent this notion was developed later on in Dedecker and Doukhan (2003). It includes numerous kinds of causal times series, for instance the strong mixing processes (see other examples in Section 3). First, for $h: \mathbb{R}^{u} \rightarrow \mathbb{R}$ an arbitrary function, with $u \in \mathbb{N}^{*}$, denote

$$
\operatorname{Lip} h=\sup _{\left(y_{1}, \ldots, y_{u}\right) \neq\left(x_{1}, \ldots, x_{u}\right)} \frac{\left|h\left(y_{1}, \ldots, y_{u}\right)-h\left(x_{1}, \ldots, x_{u}\right)\right|}{\left|y_{1}-x_{1}\right|+\cdots+\left|y_{u}-x_{u}\right|} .
$$

The time series $X=\left(X_{n}\right)_{n \in \mathbb{Z}}$ is said $\theta$-weakly dependent when there exists a sequence $\left(\theta_{r}\right)_{r \in \mathbb{N}}$ converging to 0 such that for all $r \in \mathbb{N}$, all function $f: \mathbb{R}^{2} \rightarrow \mathbb{R}$ satisfying $\|f\|_{\infty} \leq 1$, and all random variable $Z \in \mathcal{B}_{0}$ such that $\|Z\|_{\infty}<1$,

$$
\left|\operatorname{Cov}\left(f\left(X_{j_{1}}, X_{j_{2}}\right), Z\right)\right| \leq 2 \cdot \operatorname{Lip} f \cdot \theta_{r} \quad \text { for all } j_{1}, j_{2} \geq r
$$

Let us denote $\|X\|_{m}=\left(\mathbb{E}|X|^{m}\right)^{1 / m}$.

Corollary 2 Let $X=\left(X_{n}\right)_{n \in \mathbb{Z}}$ a $\theta$-weakly dependent process satisfying Assumption $M$. We also suppose that there exists $m>4$ such that $\left\|X_{0}\right\|_{m}<\infty$ and $\sum_{k=0}^{\infty} \theta_{k}^{\frac{m-4}{m-1}}<\infty$. Then the UCLT (马) holds.

\subsubsection{UCLT for non-causal weakly dependent time series}

¿From the seminal paper of Doukhan and Louhichi (1999), a second class of weakly dependent processes can be considered. This class includes also non causal time series. A process $X=\left(X_{n}\right)_{n \in \mathbb{Z}}$ with values in $\mathbb{R}^{d}$ is a so-called $\eta$-weakly dependent process if there exists a sequence $\left(\eta_{r}\right)_{r \in \mathbb{N}}$ converging to 0 , satisfying

$$
\left|\operatorname{Cov}\left(g_{1}\left(X_{i_{1}}, \ldots, X_{i_{u}}\right), g_{2}\left(X_{j_{1}}, \ldots, X_{j_{v}}\right)\right)\right| \leq\left(u\left(\operatorname{Lip} g_{1}\right)\left\|g_{2}\right\|_{\infty}+v\left(\operatorname{Lip} g_{2}\right)\left\|g_{1}\right\|_{\infty}\right) \eta_{r}
$$

for all $\left\{\begin{array}{l}\bullet(u, v) \in \mathbb{N}^{*} \times \mathbb{N}^{*} ; \\ \bullet\left(i_{1}, \ldots, i_{u}\right) \in \mathbb{Z}^{u} \text { and }\left(j_{1}, \ldots, j_{v}\right) \in \mathbb{Z}^{v} \text { with } i_{1} \leq \cdots \leq i_{u}<i_{u}+r \leq j_{1} \leq \cdots \leq j_{v} \\ \bullet \text { functions } g_{1}: \mathbb{R}^{u d} \rightarrow \mathbb{R} \text { and } g_{2}: \mathbb{R}^{v d} \rightarrow \mathbb{R} \text { satisfying } \\ \left\|g_{1}\right\|_{\infty} \leq \infty,\left\|g_{2}\right\|_{\infty} \leq \infty, \operatorname{Lip} g_{1}<\infty \text { and Lip } g_{2}<\infty ;\end{array}\right.$

As a particular case of the functional limit theorem presented in Bardet et al. (2005) a UCLT for integrated periodogram can also be established, and more precisely a convergence rate to the Gaussian law

Theorem 3 Let $X=\left(X_{n}\right)_{n \in \mathbb{Z}}$ be $\eta$-weakly dependent process and such that assumption M holds. Suppose also that

$$
\exists m>4 \text {, such that }\left\|X_{0}\right\|_{m}<\infty \text { and } \eta_{n}=\mathcal{O}\left(n^{-\alpha}\right) \text { with } \alpha>\max \left(3 ; \frac{2 m-1}{m-4}\right) .
$$

Then the UCLT (马) holds. Moreover, for $\phi: \mathbb{R} \rightarrow \mathbb{R}$ a $\mathcal{C}^{3}(\mathbb{R})$ function having bounded derivatives up to order 3 , and for $g \in \mathcal{H}_{s}$

$$
\left|\mathbb{E}\left[\phi\left(\sqrt{n}\left(J_{n}(g)-J(g)\right)\right)-\phi(\gamma(g) \cdot N)\right]\right| \leq C \cdot n^{-\frac{t}{t+3}\left(\frac{\alpha(m-4)-2 m+1}{2(m+1+\alpha \cdot m)}\right)}
$$

where $C>0, t=\left(\left(2 \alpha \frac{m-2}{m-1}-1\right) \wedge\left(s-\frac{1}{2}\right)\right), N \sim \mathcal{N}(0,1)$ and $\gamma^{2}(g)=\Gamma(g, g)$ defined in (5).

Corollary 3 Under the same assumptions as in Theorem $\mathbb{S}$, for $\ell \in \mathbb{Z}$ and $\phi: \mathbb{R} \rightarrow \mathbb{R}$ a $\mathcal{C}^{3}(\mathbb{R})$ function having bounded derivatives up to order 3 ,

$$
\left|\mathbb{E}\left[\phi\left(\sqrt{n}\left(\widehat{R}_{n}(\ell)-R(\ell)\right)\right)-\phi\left(\sigma_{\ell} \cdot N\right)\right]\right| \leq C \cdot n^{-\frac{\alpha(m-4)-2 m+1}{2(m+1+\alpha \cdot m)}}
$$

with $C>0, N \sim \mathcal{N}(0,1)$ and

$$
\left.\sigma_{\ell}^{2}=4 \pi \int_{-\pi}^{\pi} \cos ^{2}(\lambda \ell)\right) f^{2}(\lambda) d \lambda+2 \pi \int_{-\pi}^{\pi} \int_{-\pi}^{\pi} \cos (\lambda \ell) \cos (\mu \ell) f_{4}(\lambda,-\mu, \mu) d \lambda d \mu .
$$


Remark The convergence rates in both the functional limit theorems in Theorem 3 and the Corollary 3 are obtained by using Bernstein's blocks method. Even if the conditions are perhaps not optimal we derive convergence rates $n^{-\lambda}$ in those theorems with $\lambda<1 / 2$. This $\lambda$ could be made as close to $1 / 2$ as one wants by taking $s$ and $\alpha$ large enough.

\section{Applications to parametric estimation}

Now we will apply the previous results to finite parameters estimates. Let $X=\left(X_{n}\right)_{n \in \mathbb{Z}}$ be a time series satisfying Assumption M. We denote by $f$ the spectral density of $X$. Define

$$
\sigma^{2}=\exp \left(\frac{1}{2 \pi} \int_{-\pi}^{\pi} \log f(\lambda) d \lambda\right)
$$

Following Rosenblatt (1985) we shall assume that the one-step prediction error variance satifies $2 \pi \sigma^{2}>0$. Assume that $f$ belongs to the family of functions defined in the form

$$
f(\lambda)=f_{\left(\beta, \sigma^{2}\right)}(\lambda)=\sigma^{2} \cdot g_{\beta}(\lambda) \text { for all } \lambda \in[-\pi, \pi[,
$$

the function $f$ thus depends on a finite number of unknown parameters, a variance term $\sigma^{2}$ and a $\mathbb{R}^{p}$-vector $\beta$, where $\beta=\left(\beta^{(1)}, \ldots, \beta^{(p)}\right)$. The normalization condition (12) implies:

$$
\int_{-\pi}^{\pi} \log g_{\beta}(\lambda) d \lambda=0
$$

Denote also $\sigma^{*}$ and $\beta^{*}=\left(\beta^{(1) *}, \ldots, \beta^{(p) *}\right)$ the true value of $\sigma$ and $\beta$. As a consequence, for all $\lambda \in[-\pi, \pi[$, we will now denote $\sigma^{* 2} g_{\beta^{*}}(\lambda)$ the spectral density of $X$. We will also consider the following conditions

- Condition $\mathrm{C} 1$ : the true values $\sigma^{*}$ and $\beta^{*}$ are such that $\sigma^{*}>0$ and $\beta^{*}$ lies in a region $\mathcal{K} \subset \mathbb{R}^{p}$ where $\mathcal{K}$ is an open and relatively compact set.

- Condition $\mathrm{C} 2$ : if $\beta_{1}, \beta_{2}$ are distinct elements of $\mathcal{K}$, the set $\left\{\lambda \in\left[-\pi, \pi\left[, g_{\beta_{1}}(\lambda) \neq g_{\beta_{2}}(\lambda)\right\}\right.\right.$ has positive Lebesgue measure.

- Condition C3: for all $\beta \in \mathcal{K}$, the function $\lambda \mapsto g_{\beta}^{-1}(\lambda)=\frac{1}{g_{\beta}(\lambda)} \in \mathcal{H}_{s}$ and $\sup _{\beta \in \mathcal{K}}\left\|g_{\beta}^{-1}\right\|_{\mathcal{H}_{s}}<\infty$ with $s>1 / 2$.

- Condition C4: for all $\lambda \in\left[-\pi, \pi\left[\right.\right.$, the function $\beta \mapsto g_{\beta}^{-1}(\lambda)$ is continuous on $\mathcal{K}$.

- Condition C5: for all $\lambda \in\left[-\pi, \pi\left[\right.\right.$, the function $\beta \mapsto g_{\beta}^{-1}(\lambda)$ is twice continuously differentiable on $\mathcal{K}$ and $\beta \mapsto \int_{-\pi}^{\pi} \log \left(g_{\beta}(\lambda)\right) d \lambda$ can be differentiated twice under the integral sign.

- Condition $\mathrm{C} 6$ : there exists $s>1 / 2$ such that for all $\beta \in \mathcal{K}$ and $(i, j) \in\{1, \ldots, p\}$, the functions $\lambda \mapsto\left(\frac{\partial g_{\beta}^{-1}}{\partial \beta^{(i)}}\right)_{\beta}(\lambda), \lambda \mapsto\left(\frac{\partial^{2} g_{\beta}^{-1}}{\partial \beta^{(i)} \partial \beta^{(j)}}\right)_{\beta}(\lambda)$ belong to $\mathcal{H}_{s}$ and $\sup _{\beta \in \mathcal{K}}\left\|\frac{\partial^{2} g_{\beta}^{-1}}{\partial \beta^{(i)} \partial \beta^{(j)}}\right\|_{\mathcal{H}_{s}}<\infty$.

- Condition $\mathrm{C} 7$ : for all $\beta \in \mathcal{K}$, the function $\lambda \mapsto g_{\beta}(\lambda)$ is continuously differentiable on $[-\pi, \pi[$.

Let $\left(X_{1}, \ldots, X_{n}\right)$ be a sample from $X$. As usually, define Whittle's maximum likelihood estimators of $\beta^{*}$ and $\sigma^{* 2}$ as:

$$
\widehat{\beta}_{n}=\operatorname{Argmin}_{\beta \in \mathcal{K}}\left\{J_{n}\left(g_{\beta}^{-1}\right)\right\}=\operatorname{Argmin}_{\beta \in \mathcal{K}}\left\{\int_{-\pi}^{\pi} \frac{I_{n}(\lambda)}{g_{\beta}(\lambda)} d \lambda\right\} \quad \text { and } \quad \widehat{\sigma}_{n}^{2}=\frac{1}{2 \pi} J_{n}\left(g_{\widehat{\beta}_{n}}^{-1}\right)
$$

( $\widehat{\beta}_{n}$ is supposed to be uniquely defined). In the following paragraphs, we will show the strong consistency of the estimators $\widehat{\beta}_{n}$ and $\widehat{\sigma}_{n}^{2}$. 


\subsection{Asymptotic properties of Whittle's parametric estimators}

Theorem 4 Let $X$ satisfy the assumptions of Theorem 1 . Under Conditions C1-4, then

$$
\widehat{\beta}_{n} \underset{n \rightarrow \infty}{\stackrel{a . s .}{\longrightarrow}} \beta^{*} \text { and } \widehat{\sigma}_{n}^{2} \underset{n \rightarrow \infty}{\stackrel{a . s .}{\longrightarrow}} \sigma^{* 2} \text {. }
$$

Proof. From Theorem 1 and Condition C3 with probability 1,

$$
\lim _{n \rightarrow \infty} J_{n}\left(g_{\beta}^{-1}\right)=J\left(g_{\beta}^{-1}\right)
$$

uniformly on $\beta \in \mathcal{K}$. From Conditions C2 and normalization condition (14),

$$
J\left(g_{\beta}^{-1}\right)>2 \pi \sigma^{* 2}=J\left(g_{\beta^{*}}^{-1}\right) \text { for all } \beta \neq \beta^{*}
$$

(see Lemma 2, in Hannan, 1973). Therefore (see the details of the proof of Theorem 1 in Hannan, 1973), $\widehat{\beta}_{n}=\operatorname{Argmin}_{\beta \in \mathcal{K}}\left\{J_{n}\left(g_{\beta}^{-1}\right)\right\}$ converges a.s. to $\beta^{*}$ and $\widehat{\sigma}_{n}^{2}=\frac{1}{2 \pi} J_{n}\left(g_{\widehat{\beta}_{n}}^{-1}\right)$ converges to $\sigma^{* 2}=\frac{1}{2 \pi} J\left(g_{\beta^{*}}^{-1}\right)$.

Remarks on the conditions C1-4 The C1 and C2 conditions are usual and can be found for example in Rosenblatt (1985) for mixing time series or in Fox and Taqqu (1986) for strongly dependent times series. The condition $\mathrm{C} 4$ is weaker than the condition of differentiability generally required. It thus necessitates the unusual condition $\mathrm{C} 3$ related to the uniform limit theorems 1 and 2 .

Theorem 5 Let $X$ satisfy either the assumptions of Theorem 0 or those of Theorem 3 . Under Conditions C1-6 and if the matrix $W^{*}=\left(w_{i j}^{*}\right)_{1 \leq i, j \leq p}$, with

$$
w_{i j}^{*}=\int_{-\pi}^{\pi} g_{\beta^{*}}^{2}(\lambda)\left(\frac{\partial g_{\beta}^{-1}}{\partial \beta^{(i)}}\right)_{\beta^{*}}(\lambda)\left(\frac{\partial g_{\beta}^{-1}}{\partial \beta^{(j)}}\right)_{\beta^{*}}(\lambda) d \lambda
$$

is nonsingular, then

$$
\sqrt{n}\left(\widehat{\beta}_{n}-\beta^{*}\right) \quad \underset{n \rightarrow \infty}{\stackrel{\mathcal{D}}{\longrightarrow}} \mathcal{N}_{p}\left(0,\left(\sigma^{*}\right)^{-4}\left(W^{*}\right)^{-1} Q^{*}\left(W^{*}\right)^{-1}\right)
$$

with a matrix $Q^{*}=\left(q_{i j}^{*}\right)_{1 \leq i, j \leq p}$ whose entries are defined by

$$
q_{i j}^{*}=2 \pi\left(2 \sigma^{* 4} w_{i j}^{*}+\int_{-\pi}^{\pi} \int_{-\pi}^{\pi} f_{4}(\lambda, \mu,-\mu)\left(\frac{\partial g_{\beta}^{-1}}{\partial \beta^{(i)}}\right)_{\beta^{*}}(\lambda)\left(\frac{\partial g_{\beta}^{-1}}{\partial \beta^{(j)}}\right)_{\beta^{*}}(\mu) d \lambda d \mu\right) .
$$

Proof. Let $U_{n}(\beta)=J_{n}\left(g_{\beta}^{-1}\right)$. From Conditions 2 and $5, \beta \mapsto U_{n}(\beta)$ exists and is twice differentiable on $\mathcal{K}$. Denote $\frac{\partial}{\partial \beta} U_{n}(\beta)$ the vector $\left(\frac{\partial}{\partial \beta^{(i)}} U_{n}(\beta)\right)_{1 \leq i \leq p}$ and $\frac{\partial^{2}}{\partial \beta^{2}} U_{n}(\beta)$ the $(p \times p)$ matrix $\left(\frac{\partial^{2}}{\partial \beta^{(i)} \partial \beta^{(j)}} U_{n}(\beta)\right)_{1 \leq i, j \leq p}$. According to the mean value theorem,

$$
\frac{\partial}{\partial \beta} U_{n}\left(\widehat{\beta}_{n}\right)=\frac{\partial}{\partial \beta} U_{n}\left(\beta^{*}\right)+\frac{\partial^{2}}{\partial \beta^{2}} U_{n}\left(\bar{\beta}_{n}\right)\left(\widehat{\beta}_{n}-\beta^{*}\right)
$$

where $\left\|\bar{\beta}_{n}-\beta^{*}\right\|_{p} \leq\left\|\widehat{\beta}_{n}-\beta^{*}\right\|_{p}$ (with $\|\cdot\|_{p}$ denoting the euclidian norm in $\mathbb{R}^{p}$ ). Since $\widehat{\beta}_{n}$ minimizes $\beta \mapsto U_{n}(\beta)$, it follows that

$$
\frac{\partial}{\partial \beta} U_{n}\left(\beta^{*}\right)=\left[-\frac{\partial^{2}}{\partial \beta^{2}} U_{n}\left(\bar{\beta}_{n}\right)\right]\left(\widehat{\beta}_{n}-\beta^{*}\right)
$$

But, from Theorem田, $\widehat{\beta}_{n} \underset{n \rightarrow \infty}{\stackrel{a . s .}{\longrightarrow}} \beta^{*}$ and then $\bar{\beta}_{n} \underset{n \rightarrow \infty}{\stackrel{a . s .}{\longrightarrow}} \beta^{*}$. Consequently, from Condition C5, C6 and Theorem 1 (Uniform Law of Large Number),

$$
\frac{\partial^{2}}{\partial \beta^{2}} U_{n}\left(\bar{\beta}_{n}\right) \underset{n \rightarrow \infty}{\stackrel{a . s .}{\longrightarrow}}\left(\int_{-\pi}^{\pi} \frac{\partial^{2}}{\partial \beta^{(i)} \partial \beta^{(j)}} g_{\beta^{*}}^{-1}(\lambda) \cdot \sigma^{* 2} g_{\beta^{*}}(\lambda) d \lambda\right)_{1 \leq i, j \leq p}=\sigma^{* 2} W^{*},
$$

(see Lemma 3 of Fox and Taqqu, 1986). Moreover, from Theorem 2 and Condition C5,

$$
\begin{array}{rrr}
\sqrt{n}\left(\frac{\partial}{\partial \beta} U_{n}\left(\beta^{*}\right)-\frac{\partial}{\partial \beta} J\left(g_{\beta^{*}}^{-1}\right)\right) & \underset{n \rightarrow \infty}{\stackrel{\mathcal{D}}{\longrightarrow}} & \mathcal{N}_{p}\left(0, Q^{*}\right), \\
\text { and thus } \sqrt{n} \frac{\partial}{\partial \beta} U_{n}\left(\beta^{*}\right) & \underset{n \rightarrow \infty}{\stackrel{\mathcal{D}}{\longrightarrow}} & \mathcal{N}_{p}\left(0, Q^{*}\right),
\end{array}
$$


because $\frac{\partial}{\partial \beta} J\left(g_{\beta^{*}}^{-1}\right)=\int_{-\pi}^{\pi}\left(\frac{\partial g_{\beta}^{-1}(\lambda)}{\partial \beta}\right)_{\beta^{*}} \cdot \sigma^{* 2} g_{\beta^{*}}(\lambda) d \lambda=\sigma^{* 2} \frac{\partial}{\partial \beta}\left(\int_{-\pi}^{\pi} \log \left(g_{\beta}^{-1}(\lambda)\right) d \lambda\right)_{\beta^{*}}=0 \quad$ from C6 and (14). Therefore, if the matrix $W^{*}$ is nonsingular, from (16),

$$
\sqrt{n}\left(\widehat{\beta}_{n}-\beta^{*}\right) \underset{n \rightarrow \infty}{\stackrel{\mathcal{D}}{\longrightarrow}}-\left(\sigma^{*}\right)^{-2}\left(W^{*}\right)^{-1} \cdot \mathcal{N}_{p}\left(0, Q^{*}\right),
$$

and this completes the proof of Theorem .

Theorem 6 Let $X$ satisfy either the assumptions of Theorem 目 or those of Theorem . Under Conditions C1-7, then

$$
\sqrt{n}\left(\widehat{\sigma}_{n}^{2}-\sigma^{*}\right) \underset{n \rightarrow \infty}{\stackrel{\mathcal{D}}{\longrightarrow}} \mathcal{N}\left(0,2 \sigma^{* 4}+2 \pi \int_{-\pi}^{\pi} \int_{-\pi}^{\pi} f_{4}(\lambda, \mu,-\mu) g_{\beta^{*}}^{-1}(\lambda) g_{\beta^{*}}^{-1}(\mu) d \lambda d \mu\right) .
$$

Moreover, $\sqrt{n}\left(\widehat{\sigma}_{n}^{2}-\sigma^{*}\right)$ and $\sqrt{n}\left(\widehat{\beta}_{n}-\beta^{*}\right)$ are jointly asymptotically normal with covariance given by

$$
\begin{aligned}
\lim _{n \rightarrow \infty} \sqrt{n}\left(\operatorname{Cov}\left(\widehat{\sigma}_{n}^{2}, \widehat{\beta}_{n}^{(i)}\right)\right)_{1 \leq i \leq p} & \\
& =\left(\sigma^{* 2} \cdot W^{*}\right)^{-1} \cdot\left(2 \pi \int_{-\pi}^{\pi} \int_{-\pi}^{\pi} f_{4}(\lambda, \mu,-\mu) g_{\beta^{*}}^{-1}(\lambda)\left(\frac{\partial}{\partial \beta^{(i)}} g_{\beta^{*}}^{-1}(\mu)\right) d \lambda d \mu\right)_{1 \leq i \leq p} .
\end{aligned}
$$

Proof. The Taylor's formula implies that

$$
U_{n}\left(\beta^{*}\right)=U_{n}\left(\widehat{\beta}_{n}\right)+\left(\beta^{*}-\widehat{\beta}_{n}\right)^{\prime} \cdot\left(\frac{\partial^{2}}{\partial \beta^{2}} U_{n}\left(\underline{\beta}_{n}\right)\right) \cdot\left(\beta^{*}-\widehat{\beta}_{n}\right),
$$

with probability 1 , and with $\left\|\underline{\beta}_{n}-\beta^{*}\right\|_{p}<\left\|\widehat{\beta}_{n}-\beta^{*}\right\|_{p}$. From previous Theorem f, it follows

$$
\sqrt{n}\left(U_{n}\left(\beta^{*}\right)-\sigma^{* 2}\right)=\sqrt{n}\left(U_{n}\left(\widehat{\beta}_{n}\right)-\sigma^{* 2}\right)+\mathcal{O}_{p}\left(n^{-1 / 2}\right) .
$$

Under condition $\mathrm{C} 7, \mathbb{E}\left(U_{n}\left(\beta^{*}\right)\right)=\sigma^{* 2}+\mathcal{O}(\log n / n)$ (see for instance Rosenblatt, 1985, p. 78) and thus $\sqrt{n}\left(U_{n}\left(\beta^{*}\right)-\mathbb{E}\left(U_{n}\left(\beta^{*}\right)\right)\right) \underset{n \rightarrow \infty}{\stackrel{\mathcal{D}}{\longrightarrow}} \mathcal{N}\left(0, \Gamma\left(g_{\beta^{*}}^{-1}, g_{\beta^{*}}^{-1}\right)\right)$ with $g_{\beta^{*}}^{-1} \in \mathcal{H}_{s}$. Therefore,

$$
\sqrt{n}\left(U_{n}\left(\widehat{\beta}_{n}\right)-\sigma^{* 2}\right) \underset{n \rightarrow \infty}{\stackrel{\mathcal{D}}{\longrightarrow}} \mathcal{N}\left(0, \Gamma\left(g_{\beta^{*}}^{-1}, g_{\beta^{*}}^{-1}\right)\right),
$$

which implies relation (17). The end of the proof follows the same arguments as in Rosenblatt (1985).

\subsection{Examples of Whittle's parametric estimates for different time series}

\section{Causal GARCH and ARCH( $(\infty)$ processes}

The famous and from now on classical $\operatorname{GARCH}\left(q^{\prime}, q\right)$ model was introduced by Engle (1982) and Bollerslev (1986) and is given by relations

$$
X_{k}=\rho_{k} \cdot \xi_{k} \quad \text { with } \rho_{k}^{2}=a_{0}+\sum_{j=1}^{q} a_{j} X_{k-j}^{2}+\sum_{j=1}^{q^{\prime}} c_{j} \rho_{k-j}^{2},
$$

where $\left(q^{\prime}, q\right) \in \mathbb{N}^{2}, a_{0}>0, a_{j} \geq 0$ and $c_{j} \geq 0$ for $j \in \mathbb{N}$ and $\left(\xi_{k}\right)_{k \in \mathbb{Z}}$ are i.i.d. random variables with zero mean (for an excellent survey about ARCH modelling, see Giraitis et al., 2005). Under some additional conditions, the GARCH model can be written as a particular case of $\mathrm{ARCH}(\infty)$ model (introduced in Robinson, 1991) that satisfies

$$
X_{k}=\rho_{k} \cdot \xi_{k} \quad \text { with } \rho_{k}^{2}=b_{0}+\sum_{j=1}^{\infty} b_{j} X_{k-j}^{2},
$$

with a sequence $\left(b_{j}\right)_{j}$ depending on the family $\left(a_{j}\right)$ and $\left(c_{j}\right)$. Different sufficient conditions can be given for obtaining a $m$-order stationary solution to (18) or (19). Notice that for both models (18) or (19), the spectral 
density is a constant. As a consequence, the idea of Whittle's estimation in the GARCH case (see Bollerslev,

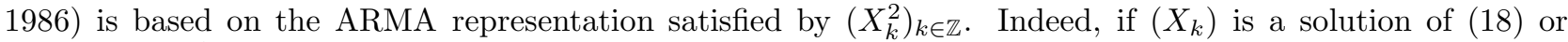
(19), then $\left(X_{k}^{2}\right)$ can be written as a solution of a particular case of equation (21) of bilinear models below (see Giraitis et al., 2005). More precisely,

$$
X_{k}^{2}=\varepsilon_{k}\left(\gamma \cdot b_{0}+\gamma \sum_{j=1}^{\infty} b_{j} X_{k-j}^{2}\right)+\lambda_{1} \cdot b_{0}+\lambda_{1} \sum_{j=1}^{\infty} b_{j} X_{k-j}^{2} \quad \text { for } k \in \mathbb{Z},
$$

with $\varepsilon_{k}=\left(\xi_{k}^{2}-\lambda_{1}\right) / \gamma$ for $k \in \mathbb{Z}, \lambda_{1}=\mathbb{E} \xi_{0}^{2}$ and $\gamma^{2}=\operatorname{Var}\left(\xi_{0}^{2}\right)$. Moreover, the time series $\left(Y_{k}\right)_{k \in \mathbb{Z}}$ defined by $Y_{k}=X_{k}^{2}-\lambda_{1} \cdot b_{0} \cdot\left(1-\lambda_{1} \sum_{j=1}^{\infty} b_{j}\right)^{-1}$ for $k \in \mathbb{Z}$, satisfies the forthcoming equation (21) with parameter $c_{0}=0$ (as in Proposition 2 below). Hence, a sufficient condition for the stationarity of $\left(X_{k}^{2}\right)_{k \in \mathbb{Z}}$ with $\left\|X_{0}^{2}\right\|_{m}<\infty$ is

$$
\left(\left\|\varepsilon_{0}\right\|_{m}+1\right) \cdot \sum_{j=1}^{\infty}\left|b_{j}\right|<1 \Longleftrightarrow\left(\frac{\left\|\xi_{0}^{2}-\lambda_{1}\right\|_{m}}{\gamma}+1\right) \cdot \sum_{j=1}^{\infty}\left|b_{j}\right|<1 .
$$

Let $\left(X_{k}\right)_{k \in \mathbb{Z}}$ a stationary solution of 19$)$ and taking $\beta=\left(\beta^{(1)}, \ldots, \beta^{(p)}\right) \in \mathbb{R}^{p}$ such that $b_{j}=b_{j}(\beta)$ for $j \in \mathbb{N}$, and $\sigma^{2}=\mathbb{E}\left(X_{0}^{2}-\rho_{0}^{2}\right)=b_{0}^{2}(\beta) \cdot h\left(\lambda_{1}, \gamma, \sum_{j=1}^{\infty} b_{j}(\beta)\right)$, where $h$ is a positive real function, the spectral density of $\left(X_{k}^{2}\right)_{k \in \mathbb{Z}}$ is

$$
f_{\left(\beta, \sigma^{2}\right)}(\lambda)=\frac{\sigma^{2}}{2 \pi} \cdot\left|1-\sum_{j=1}^{\infty} b_{j}(\beta) \cdot e^{i j \lambda}\right|^{-2}
$$

Then a Whittle's estimate can be used for estimating $\beta$ and $\sigma^{2}$ parameters in $\operatorname{ARCH}(\infty)$ process:

Proposition 1 Let $X$ be a stationary $A R C H(\infty)$ time series following equation (19), such that there exists $m>8$ satisfying $\mathbb{E}\left(\left|\xi_{0}\right|^{m}\right)<\infty$, satisfying the stationarity condition,

$$
\left(\left(\frac{\left\|\xi_{0}^{2}-\lambda_{1}\right\|_{m / 2}}{\left\|\xi_{0}^{2}-\lambda_{1}\right\|_{2}}+1\right) \wedge\left\|\xi_{0}\right\|_{m}^{2}\right) \cdot \sum_{j=1}^{\infty}\left|b_{j}(\beta)\right|<1, \text { and one of the two following assumptions }
$$

- Geometric decay: $\forall j \in \mathbb{N}, 0 \leq b_{j}(\beta)$ and $\left.\exists \mu \in\right] 0,1\left[\right.$ such that $b_{j}(\beta)=\mathcal{O}\left(\mu^{-j}\right)$;

- Riemannian decay: $\forall j \in \mathbb{N}, b_{j}(\beta) \geq 0, \exists \nu>\frac{2 m-9}{m-8}$ such that $b_{j}(\beta)=\mathcal{O}\left(j^{-\nu}\right)$.

Then, under Conditions C1-7, the central limit theorems (15) and (17) holds.

Corollary 4 If there exists $m>8$ such that $X$ is a m-order stationary $G A R C H(q, q)$ time series satisfying equation (18), then with $\beta=\left(a_{1}, \ldots, a_{q}, c_{1}, \ldots, c_{q^{\prime}}\right)$, the central limit theorems (15) and (17) are satisfied.

Proof. First, Doukhan et al (2005) have shown that $\operatorname{ARCH}(\infty)$ processes satisfy the $\theta$-weak dependence property: in the "Geometric decay" case, $\theta_{r}=\mathcal{O}\left(e^{-c \sqrt{r}}\right)$ with $c>0$ and in the "Riemannian decay" case, with $\nu>2, \theta_{r}=\mathcal{O}\left(r^{-\nu+1}\right)$. Now by applying the following Lemma (see section 5) for $h(x)=x^{2}$ and thus $a=2$, we deduce that $\left(X_{k}^{2}\right)_{k \in \mathbb{Z}}$ is a $\theta^{\frac{m-2}{m-1}}$-weak dependent time series. Hence, by denoting $\theta^{\prime}=\left(\theta_{k}^{\prime}\right)_{k \in \mathbb{Z}}$ the weak dependent sequence of $X^{2}$ it holds: in "Geometric decay" case $\theta_{r}^{\prime}=\mathcal{O}\left(e^{-c \sqrt{r}}\right)$ with $c>0$ and in the "Riemannian decay" case, $\theta_{r}^{\prime}=\mathcal{O}\left(r^{-\frac{(\nu-1)(m-2)}{m-1}}\right)$. Corollary 2 implies that $1 /$ in the "Geometric decay" case, for all $\mu$, $\left(X_{k}^{2}\right)_{k \in \mathbb{Z}}$ satisfies the Uniform CLT (9), 2 / in the "Riemannian decay" case, $\left(X_{k}^{2}\right)_{k \in \mathbb{Z}}$ satisfies the Uniform CLT (9) if $\frac{(\nu-1)(m-2)}{m-1} \cdot \frac{m / 2-4}{m / 2-1}>1$, i.e. $\nu>\frac{2 m-9}{m-8}$.

Finally, for $\operatorname{GARCH}\left(q^{\prime}, q\right)$ the spectral density is explicit $f_{\left(\beta, \sigma^{2}\right)}(\lambda)=\frac{\sigma^{2}}{2 \pi} \frac{\left|1-\Sigma_{j=1}^{q} c_{j} e^{i j \lambda}\right|^{2}}{\left|1-\Sigma_{j=1}^{q} a_{j} e^{i j \lambda}-\Sigma_{j=1}^{q} c_{j} e^{i j \lambda}\right|^{2}}$ and satisfies conditions C1-7 (more precisely conditions C3, C6 and C7 using $\mathcal{H}_{s}$ with $s>1 / 2$ are checked). Then, the proof of the corollary is a special case of the "Geometric decay" case. 
Remark Zaffaroni (2003) studied the question of the Whittle's estimation of the parameter of a stationary solution of (18), this result was improved later on by Giraitis and Robinson (2001). In this paper, the conditions to assure the asymptotic normality of the Whittle's estimate are better than our Theorem (1), in two senses: $1 /$ only the $m=8$ is required; $2 /$ the conditions on the sequence $\left(b_{j}(\beta)\right)$ in the general case of $\operatorname{ARCH}(\infty)$ model are only $b_{0}(\beta)>0$ and $b_{j}(\beta) \geq 0$ for $j \in \mathbb{N}^{*}$ and the stationarity condition $\left\|\xi_{0}\right\|_{m}^{2} \cdot \sum_{j=1}^{\infty}\left|b_{j}(\beta)\right|<1$. However, their method for establishing the central limit theorem for the periodogram is essentially ad hoc and can not be used for non causal or non linear time series. The recent book of Straumann (2005) also provides an up-to-date and complete overview to this question. Chapter 8 of this book exposed the results of Mikosch and Straumann (2002) that considered the case of intermediate moment conditions of order $>4$ and $<8$ only for special case of $\operatorname{GARCH}(1,1)$ processes. The convergence rates are slower than the present ones.

\section{Causal Bilinear processes}

Now, assume that $X=\left(X_{k}\right)_{k \in \mathbb{Z}}$ is a bilinear process (see the seminal paper of Giraitis and Surgailis, 2002) satisfying the equation

$$
X_{k}=\xi_{k}\left(a_{0}+\sum_{j=1}^{\infty} a_{j} X_{k-j}\right)+c_{0}+\sum_{j=1}^{\infty} c_{j} X_{k-j} \quad \text { for } k \in \mathbb{Z},
$$

where $\left(\xi_{k}\right)_{k \in \mathbb{Z}}$ are i.i.d. random variables with zero mean and such that $\left\|\xi_{0}\right\|_{m}<+\infty$ with $m \geq 1$, and $a_{j}, c_{j}$, $j \in \mathbb{N}$ are real coefficients. Assume $c_{0}=0$ and define the generating functions

$$
\begin{array}{ll}
A(z)=\sum_{j=1}^{\infty} a_{j} z^{j} & C(z)=\sum_{j=1}^{\infty} c_{j} z^{j} \\
G(z)=(1-C(z))^{-1}=\sum_{j=0}^{\infty} g_{j} z^{j} & H(z)=A(z) G(z)=\sum_{j=1}^{\infty} h_{j} z^{j} .
\end{array}
$$

If $\left\|\xi_{0}\right\|_{m} \cdot \sum_{j=1}^{\infty}\left|h_{j}\right|<\infty$, for instance when $\left\|\xi_{0}\right\|_{m} \cdot\left(\sum_{j=1}^{\infty}\left|a_{j}\right|+\sum_{j=1}^{\infty}\left|c_{j}\right|\right)<1$ (see Giraitis and Surgailis, 2002 ), there exists a unique zero mean stationary and ergodic solution $X$ in $\mathbb{L}^{m}(\Omega, \mathcal{A}, \mathbb{P})$ of equation (21) (see Doukhan et al., 2006). For $m \geq 2$, the covariogram of $X$ is $R(k)=a_{0}^{2} \cdot\left\|\xi_{0}\right\|_{2} \cdot\left(1-\sum_{j=1}^{\infty} h_{j}^{2}\right)^{-1} \sum_{j=0}^{\infty} g_{j} g_{j+k}$ and satisfies $\sum_{k}|R(k)|<\infty$. If we assume that there exists $\beta=\left(\beta^{(1)}, \ldots, \beta^{(p)}\right)$ such that for all $k \in \mathbb{Z}$, $a_{k}=a_{k}(\beta)$ and $c_{k}=c_{k}(\beta)$, the spectral density of $X$ exists and satisfies

$$
f_{\left(\beta, \sigma^{2}\right)}(\lambda)=\frac{a_{0}^{2}(\beta) \cdot \sigma^{2}}{2 \pi\left(1-\sum_{j=1}^{\infty} h_{j}^{2}(\beta)\right)} \sum_{k=-\infty}^{\infty} \sum_{j=0}^{\infty} g_{j}(\beta) g_{j+k}(\beta) e^{-i k \lambda},
$$

with $\sigma^{2}=\left\|\xi_{0}\right\|_{2}^{2}$. Like in Doukhan et al. (2006), we consider three different cases of the convergence rate to zero of the sequences $\left(a_{k}\right)$ and $\left(c_{k}\right)$. Then, using the previous results for $\theta$-weak dependent time series, Whittle's estimate of parameters $\beta$ and $\sigma^{2}$ satisfies the following proposition

Proposition 2 Let $X$ be a stationary bilinear time series satisfying equation (2T) with $c_{0}=0, \mathbb{E}\left(\left|\xi_{0}\right|^{m}\right)<\infty$ with $m>4$ and such that $\left\|\xi_{0}\right\|_{m} \cdot\left(\sum_{j=1}^{\infty}\left|a_{j}\right|+\sum_{j=1}^{\infty}\left|c_{j}\right|\right)<1$. Moreover, assume that $X$ satisfies one of the 3 following conditions

- Finite case: $\exists J \in \mathbb{N}$ such that $\forall j>J, a_{j}(\beta)=c_{j}(\beta)=0$;

- Geometric decay: $\exists \mu \in] 0,1\left[\right.$ such that $\sum_{j}\left|c_{j}(\beta)\right| \mu^{-j} \leq 1$ and $\forall j \in \mathbb{N}, 0 \leq a_{j}(\beta) \leq \mu^{j}$;

- Riemannian decay: $\forall j \in \mathbb{N}, c_{j}(\beta) \geq 0$, and $\exists \nu_{1}>\frac{2 m-5}{m-4}$ such that $a_{j}(\beta)=\mathcal{O}\left(j^{-\nu_{1}}\right)$ and $\exists \nu_{2}>0$ such that $\sum_{j} c_{j}(\beta) j^{1+\nu_{2}}<\infty$, with $\left\{\begin{array}{l}\nu_{2}>\frac{(m-4) \delta}{(m-1) \delta-(m-4) \log 2} \\ \delta=\log \left(1+\frac{1-\sum_{j}\left|c_{j}(\beta)\right|}{\sum_{j} c_{j}(\beta) j^{1+\nu_{2}}}\right)>\log 2 \frac{(m-4)}{(m-1)}\end{array}\right.$.

Then, under Conditions C1-7, the central limit theorems (15) and (17) holds.

Proof. Doukhan et al. (2006) studied the three different cases of the Proposition and deduced the $\theta$-weak dependence behavior of $X$ in each case. In the "Finite" and the "Geometric decay" cases, $\theta_{r}=\mathcal{O}\left(e^{-c \sqrt{r}}\right)$ with $c>0$, which implies the conditions required in Corollary 2. Therefore, under Conditions C1-7, the models satisfy the central limit theorems (15) and (17). 
In the "Riemannian decay" case, $\theta_{r}=\mathcal{O}\left(\left(\frac{r}{\log r}\right)^{d}\right)$ with $d=\max \left(-\left(\nu_{1}-1\right) ;-\frac{\nu_{2} \cdot \delta}{\delta+\nu_{2} \cdot \log 2}\right)$. The CLT (9) holds, from Corollary 2, whenever $d \cdot \frac{m-1}{m-4}<-1$. Thus, if $1-\nu_{1}<-\frac{m-4}{m-1}$ and $-\frac{\nu_{2} \cdot \delta}{\delta+\nu_{2} \cdot \log 2}<-\frac{m-4}{m-1}$, i.e. $\quad \nu_{1}>\frac{2 m-5}{m-4}$ and $\nu_{2}>\frac{(m-4) \delta}{(m-1) \delta-(m-4) \log 2}$, the assumptions of Corollary 2 hold true, and under Conditions C1-7, the central limit theorems (15) and (17) are also satisfied.

\section{Non-causal (two-sided) linear processes}

Let $X$ be a zero mean stationary non causal (two-sided) linear time series satisfying

$$
X_{k}=\sum_{j=-\infty}^{\infty} a_{j} \xi_{k-j} \text { for } k \in \mathbb{Z}
$$

with $\left(a_{k}\right)_{k \in \mathbb{Z}} \in \mathbb{R}^{\mathbb{Z}}$ and $\left(\xi_{k}\right)_{k \in \mathbb{Z}}$ a sequence of zero mean i.i.d. random variables such that $\mathbb{E}\left(\xi_{0}^{2}\right)=\sigma^{2}<\infty$ and $\mathbb{E}\left(\left|\xi_{0}\right|^{m}\right)<\infty$ with $m \geq 4$. We assume that there exists $\beta=\left(\beta^{(1)}, \ldots, \beta^{(p)}\right)$ such that for all $k \in \mathbb{Z}, a_{k}=a_{k}(\beta)$. Moreover, we assume that $\left(a_{k}(\beta)\right)_{k \in \mathbb{Z}}$ is such that $a_{k}(\beta)=\mathcal{O}\left(|k|^{-a}\right)$ with $a>1$. Therefore the spectral density of $X$ exists and satisfies

$$
f_{\left(\beta, \sigma^{2}\right)}(\lambda)=\frac{\sigma^{2}}{2 \pi}\left|\sum_{k=-\infty}^{\infty} a_{k}(\beta) e^{-i k \lambda}\right|^{2}
$$

Then the results of the previous paragraph concerning non causal weak dependent processes can be applied.

Proposition 3 Let $X$ be a linear time series satisfying (29) with $\left(a_{k}(\beta)\right)_{k \in \mathbb{Z}} \in \mathbb{R}^{\mathbb{Z}}$ and $\left(\xi_{k}\right)_{k \in \mathbb{Z}}$ a sequence of i.i.d. random variables with zero mean such that $\mathbb{E}\left(\xi_{0}^{2}\right)=\sigma^{2}$ and $\mathbb{E}\left(\left|\xi_{0}\right|^{m}\right)<\infty$ with $m>4$. We assume that $\left(a_{k}(\beta)\right)$ is such that

$$
a_{k}(\beta)=\mathcal{O}\left(|k|^{-a}\right) \quad \text { with } \quad a>\max \left\{\frac{7}{2} ; \frac{5 m-6}{2(m-4)}\right\} .
$$

Then, under Conditions C1-7, the central limit theorems (15) and 117) are satisfied.

Proof. A $\eta$-weak dependence condition for non causal linear random fields could be found in Doukhan and Lang (2002, p. 3); under the previous assumptions, $X$ is a $\eta$-weak dependent time series with the relation: $\eta_{2 r}^{2}=\mathcal{O}\left(\sum_{|k|>r} a_{k}^{2}(\beta)\right) \Longrightarrow \eta_{r}=\mathcal{O}\left(\frac{1}{r^{a-1 / 2}}\right)$. Proposition 3 is then a consequence of Theorem 3 .

Remark 1/ The Condition C7 of central limit theorem (17) is automatically satisfied by the convergence rate of $\left(a_{k}\right)$ and therefore is not required in Proposition 3.

2/The especial case of ARMA process i.e. $f_{\left(\beta, \sigma^{2}\right)}(\lambda)=\left|\frac{A\left(e^{i \lambda}\right)}{B\left(e^{i \lambda}\right)}\right|^{2}$, with $A$ and $B$ polynomials

$$
A(z)=\sum_{k=0}^{q} a_{k} z^{k}, \quad a_{0} \neq 0, \quad B(z)=\sum_{k=0}^{p} b_{k} z^{k}, \quad b_{0}=1,
$$

without zeros in the circle and with no common factors, is interesting. We have $\beta=\left(a_{0}, \ldots, a_{q}, b_{0}, \ldots, b_{p}\right)$ (Rosenblatt, 1985 page 206) and $\sigma^{2}$ could be computed by using Jessen formula's. A factorization as in (13) is then possible. In this setting conditions $\mathrm{C} 1-7$ are readily checked.

3/ To our knowledge, the known results about asymptotic behavior of Whittle's parametric estimators for non-gaussian linear processes are essentially devoted to one-sided (causal) linear processes (see for instance, Hannan, 1973, Hall and Heyde, 1980, Rosenblatt, 1985, Brockwell and Davis, 1988). In such a case, the conditions on $\left(a_{k}\right)$ are Conditions C1-6, with: $\sum_{k} k a_{k}^{2}<\infty$ for the UCLT and the existence of $\sum_{k} k a_{k} e^{-i k \lambda}$ for Condition C7. It holds true if $m=4$ and $a_{k}=\mathcal{O}\left(|k|^{-a}\right)$ with $a>2$.

4/ There exist very few results in the case of two-sided linear processes. In Rosenblatt (2000, p. 52) a condition for strong mixing property for two-sided linear processes was given, but some restrictive conditions on the process were also required for obtaining a central limit theorem for Whittle's estimators: the distribution of random variables $\xi_{k}$ has to be absolutely continuous with respect to the Lebesgue measure with a bounded variation density, $m>4+2 \delta$ with $\delta>0$ and the central limit theorem is obtained by using a tapered periodogram, under assumption $\sum_{m=1}^{\infty} \alpha_{4, \infty}(m)^{\delta /(2+\delta)}<\infty$. Here $\alpha_{4, \infty}(m) \geq \alpha_{m}$ denotes a strong mixing 
coefficient defined now with four points in the future instead of 2 for $\alpha_{m}^{\prime}$, the same remark following Corollary 1 still holds. The case of strongly dependent two-sided linear processes was also treated by Giraitis and Surgailis (1990) or Horvath and Shao (1999), however with more restrictive conditions than Conditions C1-6 and with $a_{k}=\mathcal{O}\left(|k|^{-a}\right)$ for a fixed $-1<a<0$.

5/ In the case of causal linear processes, it is well known that

$$
\sqrt{n}\left(\widehat{\beta}_{n}-\beta^{*}\right) \underset{n \rightarrow \infty}{\stackrel{\mathcal{D}}{\longrightarrow}} \mathcal{N}_{p}\left(0,2 \pi \cdot\left(W^{*}\right)^{-1}\right),
$$

$\widehat{\sigma}_{n}^{2}$ is a consistent estimate of $\sigma^{*}$, and $\sqrt{n}\left(\widehat{\beta}_{n}-\beta^{*}\right)$ and $\sqrt{n}\left(\widehat{\sigma}_{n}^{2}-\sigma^{*}\right)$ are asymptotically normal and independent.

\section{Non-causal Volterra processes}

A zero mean and non causal process $X=\left(X_{t}\right)_{t \in \mathbb{Z}}$ is called a non-causal Volterra process, if it satisfies

$$
X_{k}=\sum_{p=1}^{\infty} Y_{k}^{(p)}, \quad \text { with } \quad Y_{k}^{(p)}=\sum_{\substack{j_{1}<j_{2}<\ldots<j_{p} \\ j_{1}, \ldots, j_{p} \in \mathbb{Z}}} a_{j_{1}, \ldots, j_{p}} \xi_{k-j_{1}} \cdots \xi_{k-j_{p}},
$$

where $\left(a_{j_{1}, \ldots, j_{p}}\right) \in \mathbb{R}$ for $p \in \mathbb{N}^{*}$ and $\left(j_{1}, \ldots, j_{p}\right) \in \mathbb{Z}^{p}$, and $\left(\xi_{k}\right)_{k \in \mathbb{Z}}$ a sequence of zero mean i.i.d. random variables such that $\mathbb{E}\left(\xi_{0}^{2}\right)=\sigma^{2}<\infty$ and $\mathbb{E}\left(\left|\xi_{0}\right|^{m}\right)<\infty$ with $m>4$. Such a Volterra process is a natural extension of the previous case of non-causal linear process. From Doukhan (2003), the existence of $X$ and thus the stationarity in $\mathbb{L}^{m}$ relies on the assumption

$$
\sum_{p=0}^{\infty} \sum_{\substack{j_{1}<j_{2}<\ldots<j_{p} \\ j_{1}, \ldots, j_{p} \in \mathbb{Z}}}\left|a_{j_{1}, \ldots, j_{p}}\right|^{m}\left\|\xi_{0}\right\|_{m}^{p}<\infty
$$

Assume that there exists $\beta=\left(\beta^{(1)}, \ldots, \beta^{(p)}\right)$ such that for all $p \in \mathbb{N}^{*}$ and $\left(j_{1}, \ldots, j_{p}\right) \in \mathbb{Z}^{p}, a_{j_{1}, \ldots, j_{p}}=$ $a_{j_{1}, \ldots, j_{p}}(\beta)$. Then the spectral density of $X$ exists and satisfies

$$
f_{\left(\beta, \sigma^{2}\right)}(\lambda)=\sum_{p=1}^{\infty} \frac{\left(\sigma^{2}\right)^{p}}{2 \pi} \sum_{k=-\infty}^{\infty} \sum_{\substack{j_{1}<j_{2}<\ldots<j_{p} \\ j_{1}, \ldots, j_{p} \in \mathbb{Z}}} a_{j_{1}, \ldots, j_{p}}(\beta) \cdot a_{j_{1}+k, \ldots, j_{p}+k}(\beta) \cdot e^{-i k \lambda}
$$

(this formula is provided by the computation of the covariances of $X$; remark that the representation with strictly ordered indices $j_{1}<j_{2}<\cdots<j_{p}$ is fundamental). Certain conditions on the asymptotic behavior of the coefficients $a_{j_{1}, \ldots, j_{p}}(\beta)$ give the $\eta$-weak dependence property of $X$ and then the asymptotic normality of estimators $\left(\widehat{\beta}_{n}, \widehat{\sigma}_{n}^{2}\right)$

Proposition 4 Let $X$ be a non-causal zero mean stationary Volterra process satisfying relation (23) where $\left(a_{j_{1}, \ldots, j_{p}}^{(p)}\right) \in \mathbb{R}$ for $p \in \mathbb{N}^{*}$ and $\left(j_{1}, \ldots, j_{p}\right) \in \mathbb{Z}^{p}$, and $\left(\xi_{k}\right)_{k \in \mathbb{Z}}$ a sequence of zero mean i.i.d. random variables such that $\mathbb{E}\left(\xi_{0}^{2}\right)=\sigma^{2}<\infty$ and $\mathbb{E}\left(\left|\xi_{0}\right|^{m}\right)<\infty$ with $m>4$.

Moreover, assume that the process is in some finite order chaos (i.e. $a_{j_{1}, \ldots, j_{p}}(\beta)=0$ for $\left.p>p_{0}\right)$ and $a_{j_{1}, \ldots, j_{p}}(\beta)$ is such that

$$
a_{j_{1}, \ldots, j_{p}}(\beta)=\mathcal{O}\left(\max _{1 \leq i \leq p}\left\{\left|j_{i}\right|^{-a}\right\}\right) \quad \text { with } \quad a>4+\max \left\{0 ; \frac{11-m}{m-4}\right\} .
$$

Then, under Conditions C1-7, the central limit theorems (15) and (17) are satisfied.

Remark A sharper dependence assumption is $\eta_{r}=\mathcal{O}\left(r^{1-a}\right)$ where $a$ is submitted to the same restriction as before; recall that

$$
\eta_{r} \leq 2 \sum_{k=1}^{\infty} \sum_{\substack{j_{1}<j_{2}<\ldots<j_{k} \\ j_{1}<-r / 2, \text { or } j_{k} \geq r / 2}}\left|a_{j_{1}, \ldots, j_{k}}^{(k)}\right|\left\|\xi_{0}\right\|_{1}^{k}<\infty
$$

Proof. From Doukhan (2003), under the previous assumptions, $X$ is a weakly dependent process with

$$
\eta_{r} \leq \sum_{p=1}^{\infty} \sum_{\substack{j_{1}<j_{2}<\ldots<j_{p} \\ j_{1}<-r / 2, \text { or } j_{p} \geq r / 2}}\left|a_{j_{1}, \ldots, j_{p}}^{(p)}\right|\left\|\xi_{0}\right\|_{1}^{p}<\infty \quad \Longrightarrow \quad \eta_{r}=\mathcal{O}\left(\frac{1}{r^{a+1}}\right) .
$$


Proposition 1 is then a consequence of Theorem 3 .

\section{Non-causal linear processes with dependent innovations}

Let $X=\left(X_{n}\right)_{n \in \mathbb{N}}$ be a zero mean stationary non causal (two-sided) linear time series satisfying equation (22) with a dependent innovation process. More precisely, let $\left(\xi_{n}\right)_{n \in \mathbb{Z}}$ be a weakly dependent fourth order centered stationary process verifying Assumption $M$ and such that $\mathbb{E}\left(\xi_{0}^{2}\right)=\sigma^{2}<\infty$. Assume that there exists $\beta=\left(\beta^{(1)}, \ldots, \beta^{(p)}\right)$ such that for all $k \in \mathbb{Z}, a_{k}=a_{k}(\beta)$ with $a_{k}(\beta)=\mathcal{O}\left(|k|^{-a}\right)$ and $a>1$. Denoting $g_{\left(\beta, \sigma^{2}\right)}$ the spectral density of the process $\left(\xi_{n}\right)_{n \in \mathbb{Z}}$, the spectral density of $X$ exists and satisfies

$$
f_{\left(\beta, \sigma^{2}\right)}(\lambda)=g_{\left(\beta, \sigma^{2}\right)}(\lambda) \cdot\left|\sum_{k=-\infty}^{\infty} a_{k}(\beta) e^{-i k \lambda}\right|^{2}
$$

For instance, the process $\left(\xi_{n}\right)_{n \in \mathbb{Z}}$ may be a causal or a non-causal $\mathrm{ARCH}(\infty)$ or bilinear process. Following the results of Doukhan and Wintenberger $(2006)$, if $\left(\xi_{n}\right)_{n \in \mathbb{Z}}$ is a $\eta$-weakly dependent process, then $X$ is an $\eta$-weakly dependent process (with a sequence $\left(\eta_{r}\right)_{r}$ that can be deduced). Thus, the asymptotic normality of the Whittle estimate of parameters $\left(\beta, \sigma^{2}\right)$ could be established

Proposition 5 Let $X$ be a linear time series satisfying (22) with $\left(a_{k}(\beta)\right)_{k \in \mathbb{Z}} \in \mathbb{R}^{\mathbb{Z}}$ and $\left(\xi_{k}\right)_{k \in \mathbb{Z}}$ a $\eta^{(\xi)}-$ weakly dependent process with zero mean, a spectral density $g_{\left(\beta, \sigma^{2}\right)}$ depending only on parameters $\left(\beta, \sigma^{2}\right)$, and such that $\mathbb{E}\left(\xi_{0}^{2}\right)=\sigma^{2}$ and $\mathbb{E}\left(\left|\xi_{0}\right|^{m}\right)<\infty$. Moreover, we assume that

$$
a_{k}(\beta)=\mathcal{O}\left(|k|^{-a}\right), \quad \eta_{r}^{(\xi)}=\mathcal{O}\left(r^{-b}\right), \quad \text { with } \quad b \cdot \frac{(a-2)(m-2)}{(a-1)(m-1)}>\max \left\{3 ; \frac{2 m-1}{m-4}\right\} .
$$

Then, under Conditions C1-7, the central limit theorems (15) and (17) are satisfied.

Proof. Doukhan and Wintenberger (2006) proved, under assumptions $a_{k}(\beta)=\mathcal{O}\left(|k|^{-a}\right)$ and $\eta_{r}^{(\xi)}=\mathcal{O}\left(r^{-b}\right)$, that $X$ is a $\eta$-weakly dependent process with $\eta_{r}=\mathcal{O}\left(r^{-b \cdot \frac{(a-2)(m-2)}{(a-1)(m-1)}}\right)$. Proposition 5 is then a consequence of Theorem 3 .

\section{Appendix: proofs}

\subsection{Proof of Theorem 1}

Lemma 2 If $X$ satisfies Assumption $M$, then

$$
n \cdot \max _{\ell \geq 0}\left(\operatorname{Var}\left(\widehat{R}_{n}(\ell)\right)\right) \leq \kappa_{4}+2 \gamma .
$$

Proof of Lemma . To prove this result, we use the identity

$$
\operatorname{Cov}\left(Y_{0, \ell}, Y_{j, \ell}\right)=\kappa_{4}(\ell, j, j+\ell)+R(j)^{2}+R(j+\ell) R(j-\ell),
$$

and deduce from the stationarity of $\left(Y_{j, \ell}\right)_{j \in \mathbb{Z}}$ when $\ell$ is a fixed integer

$$
\begin{aligned}
n \cdot \operatorname{Var}\left(\widehat{R}_{n}(\ell)\right) & \leq \frac{1}{n} \sum_{j=1 \vee(1-\ell)}^{(n-\ell) \wedge n} \sum_{j^{\prime}=1 \vee(1-\ell)}^{(n-\ell) \wedge n}\left|\operatorname{Cov}\left(Y_{j, \ell}, Y_{j^{\prime}, \ell}\right)\right| \\
& \leq \sum_{j \in \mathbb{Z}}\left|\operatorname{Cov}\left(Y_{0, \ell}, Y_{j, \ell}\right)\right| \\
& \leq \sum_{j \in \mathbb{Z}}\left(\left|\kappa_{4}(\ell, j, j+\ell)\right|+2 R(j)^{2}\right) \\
& \leq \kappa_{4}+2 \gamma,
\end{aligned}
$$

by using Cauchy-Schwarz inequality for $\ell^{2}$-sequences.

Lemma 3 If $X$ satisfies Assumption $M$, then

$$
\mathbb{E}\left\|J_{n}-J\right\|_{\mathcal{H}_{s}^{\prime}}^{2} \leq \frac{3}{n}\left(\gamma+c_{s} \cdot\left(\kappa_{4}+2 \gamma\right)\right), \quad \text { where } c_{s} \text { is defined in (2). }
$$


Proof of Lemma S. Let $g(\lambda)=\sum_{\ell \in \mathbb{Z}} g_{\ell} e^{i \ell \lambda} \in \mathcal{H}_{s}$. As in Doukhan and León (1989), we use the decomposition

$$
J_{n}(g)-J(g)=-T_{1}(g)-T_{2}(g)+T_{3}(g) \text { with }\left\{\begin{array}{l}
T_{1}(g)=\sum_{|\ell| \geq n} R(\ell) g_{\ell}, \\
T_{2}(g)=\frac{1}{n} \sum_{|\ell|<n}|\ell| R(\ell) g_{\ell}, \\
T_{3}(g)=\sum_{|\ell|<n}\left(\widehat{R}_{n}(\ell)-\mathbb{E} \widehat{R}_{n}(\ell)\right) g_{\ell}
\end{array}\right.
$$

Remark that $T_{3}(g)=J_{n}(g)-\mathbb{E} J_{n}(g)$. Thus, we obtain the inequality

$$
\mathbb{E}\left\|J_{n}-J\right\|_{\mathcal{H}_{s}^{\prime}}^{2} \leq 3\left(\left\|T_{1}\right\|_{\mathcal{H}_{s}^{\prime}}^{2}+\left\|T_{2}\right\|_{\mathcal{H}_{s}^{\prime}}^{2}+\mathbb{E}\left\|T_{3}\right\|_{\mathcal{H}_{s}^{\prime}}^{2}\right) .
$$

Cauchy-Schwarz inequality yields

$$
\begin{aligned}
\left\|T_{1}\right\|_{\mathcal{H}_{s}^{\prime}}^{2} & \leq \sum_{|\ell| \geq n}(1+|\ell|)^{-2 s} R(\ell)^{2} \leq \frac{1}{n} \sum_{|\ell| \geq n} R(\ell)^{2}, \\
\left\|T_{2}\right\|_{\mathcal{H}_{s}^{\prime}}^{2} & \leq \frac{1}{n^{2}} \sum_{|\ell|<n}|\ell|^{2}(1+|\ell|)^{-2 s} R(\ell)^{2} \leq \frac{1}{n} \sum_{|\ell|<n} R(\ell)^{2} .
\end{aligned}
$$

Hence, $\left\|T_{1}\right\|_{\mathcal{H}_{s}^{\prime}}^{2}+\left\|T_{2}\right\|_{\mathcal{H}_{s}^{\prime}}^{2} \leq \frac{\gamma}{n}$. Lemma 2 entails

$$
\begin{aligned}
\left\|T_{3}\right\|_{\mathcal{H}_{s}^{\prime}}^{2} & \leq \sum_{|\ell|<n}(1+|\ell|)^{-2 s}\left(\widehat{R}_{n}(\ell)-\mathbb{E} \widehat{R}_{n}(\ell)\right)^{2}, \\
\mathbb{E}\left\|T_{3}\right\|_{\mathcal{H}_{s}^{\prime}}^{2} & \leq \sum_{|\ell|<n}(1+|\ell|)^{-2 s} \operatorname{Var}\left(\widehat{R}_{n}(\ell)\right) \leq \frac{1}{n} \sum_{|\ell|<n}(1+|\ell|)^{-2 s}\left(\kappa_{4}+2 \gamma\right) \leq \frac{c_{s}\left(\kappa_{4}+2 \gamma\right)}{n},
\end{aligned}
$$

with $c_{s}$ defined in (2). We combine those results to deduce Lemma 3 .

Proof of Theorem 日. We prove this strong law of large numbers from a weak $\mathbb{L}^{2}$-LLN and Lemma 3. The scheme of proof is analogue to the one in the standard strong LLN in $\mathbb{L}^{2}$. Set $t>0$. First, we know that for all random variables $X$ and $Y$, we have $\mathbb{P}(X+Y \geq 2 t) \leq \mathbb{P}(X \geq t)+\mathbb{P}(Y \geq t)$. Thus

$$
\begin{aligned}
\mathbb{P}\left(\sup _{n \geq N}\left\|J_{n}-J\right\|_{\mathcal{H}_{s}^{\prime}} \geq 2 t\right) & \leq \sum_{k=[\sqrt{N}]}^{\infty} \mathbb{P}\left(\left\|J_{k^{2}}-J\right\|_{\mathcal{H}_{s}^{\prime}} \geq t\right) \\
& +\sum_{k=[\sqrt{N}]}^{\infty} \mathbb{P}\left(\sup _{k^{2} \leq n<(k+1)^{2}}\left\|J_{n}-J_{k^{2}}\right\|_{\mathcal{H}^{\prime}} \geq t\right) \\
& \leq A_{N}+B_{N} .
\end{aligned}
$$

¿From Bienaymé-Tchebychev inequality, Lemma 3 implies that

$$
A_{N} \leq \frac{C_{1}}{t^{2}} \cdot \sum_{k \geq \sqrt{N}} \frac{1}{k^{2}}
$$

with $C_{1} \in \mathbb{R}_{+}$. Now set $\widetilde{R}_{n}(\ell)=\widehat{R}_{n}(\ell)-\mathbb{E} \widehat{R}_{n}(\ell)$. The fluctuation term $B_{N}$ is more involved and its bound is based on the same type of decomposition as (24), because for $k^{2}<n$

$$
J_{n}(g)-J_{k^{2}}(g)=-T_{1}^{\prime}(g)+T_{2}^{\prime}(g)-T_{3}^{\prime}(g),
$$

$$
\begin{aligned}
\text { with now } T_{1}^{\prime}(g) & =\sum_{k^{2} \leq|\ell|<n} R(\ell) g_{\ell}, \\
T_{2}^{\prime}(g) & =\frac{1}{n} \sum_{k^{2} \leq|\ell|<n}|\ell| R(\ell) g_{\ell}, \\
\text { and } T_{3}^{\prime}(g) & =\sum_{|\ell|<k^{2}} \widetilde{R}_{k^{2}}(\ell) g_{\ell}-\sum_{|\ell|<n} \widetilde{R}_{n}(\ell) g_{\ell} .
\end{aligned}
$$


As previously, $\left\|T_{1}^{\prime}\right\|_{\mathcal{H}_{s}^{\prime}}^{2}+\left\|T_{2}^{\prime}\right\|_{\mathcal{H}_{s}^{\prime}}^{2} \leq \frac{\gamma}{k^{2}}$.

Set $L_{k}=\max _{k^{2} \leq n<(k+1)^{2}}\left\|J_{n}-J_{k^{2}}\right\|_{\mathcal{H}_{s}^{\prime}}$ and $T_{k}^{*}=\max _{k^{2} \leq n<(k+1)^{2}}\left\|T_{3}^{\prime}\right\|_{\mathcal{H}_{s}^{\prime}}$. Then,

$$
B_{N} \leq \sum_{k \geq \sqrt{N}} b_{k}, \quad \text { with } \quad b_{k}=\mathbb{P}\left(L_{k} \geq t\right) \leq \frac{\mathbb{E}\left(L_{k}^{2}\right)}{t^{2}} .
$$

Now

$$
\mathbb{E}\left(L_{k}^{2}\right) \leq 3\left(\left\|T_{1}^{\prime}\right\|_{\mathcal{H}_{s}^{\prime}}^{2}+\left\|T_{2}^{\prime}\right\|_{\mathcal{H}_{s}^{\prime}}^{2}+\mathbb{E}\left\|T_{k}^{*}\right\|_{\mathcal{H}_{s}^{\prime}}^{2}\right) \leq \frac{3 \gamma}{k^{2}}+3 \cdot \mathbb{E}\left\|T_{k}^{*}\right\|_{\mathcal{H}_{s}^{\prime}}^{2}
$$

Then, for $k^{2} \leq n<(k+1)^{2}$ and $\ell \in \mathbb{Z}$,

$$
\begin{aligned}
\widetilde{R}_{n}(\ell) & =\frac{k^{2}}{n} \widetilde{R}_{k^{2}}(\ell)+\Delta_{\ell, n, k} \\
\Delta_{\ell, n, k} & =\frac{1}{n} \sum_{\left.h=k^{2} \wedge\left(k^{2}-\ell\right)\right)+1}^{n \wedge(n-\ell)}\left(X_{h} X_{h+\ell}-R(\ell)\right)=\frac{1}{n} \sum_{\left.h=k^{2} \wedge\left(k^{2}-\ell\right)\right)+1}^{n \wedge(n-\ell)} Y_{h, \ell} .
\end{aligned}
$$

Remark that $\widetilde{R}_{k^{2}}(\ell)=0$ if $k^{2} \leq|\ell| \leq n$ and thus $\widetilde{R}_{n}(\ell)=\Delta_{\ell, n, k}$ in such a case. Also note that

$$
\begin{aligned}
\Delta_{\ell, k}^{*} & =\max _{k^{2} \leq n<(k+1)^{2}}\left|\Delta_{\ell, n, k}\right| \leq \frac{1}{k^{2}} \sum_{h=\left(k^{2} \wedge\left(k^{2}-\ell\right)\right)+1}^{\left(k^{2}+2 k\right) \wedge\left(\left(k^{2}+2 k\right)-\ell\right)}\left|Y_{h, \ell}\right| \\
\text { and thus } \mathbb{E}\left(\Delta_{\ell, k}^{*}\right)^{2} & \leq \frac{1}{k^{4}}(2 k)^{2} \cdot \max _{(h, \ell) \in \mathbb{Z}^{2}}\left(\mathbb{E}\left(\left|Y_{h, \ell}\right|^{2}\right)\right) \\
& \leq \frac{4}{k^{2}} \mathbb{E}\left(\left|X_{0}\right|^{4}\right) .
\end{aligned}
$$

Write

$$
\begin{aligned}
T_{3}^{\prime}(g) & =\sum_{|\ell|<k^{2}} \widetilde{R}_{k^{2}}(\ell)\left(1-\frac{k^{2}}{n}\right) g_{\ell}-\sum_{|\ell|<n} \Delta_{\ell, n, k} g_{\ell} \\
\left|T_{k}^{*}(g)\right| & \leq \frac{2}{k} \sum_{|\ell|<k^{2}}\left|\widetilde{R}_{k^{2}}(\ell) g_{\ell}\right|+\sum_{|\ell|<(k+1)^{2}} \Delta_{\ell, k}^{*}\left|g_{\ell}\right|,
\end{aligned}
$$

and we thus deduce

$$
\mathbb{E}\left\|T_{k}^{*}\right\|_{\mathcal{H}_{s}^{\prime}}^{2} \leq 2 c \cdot\left(\frac{4}{k^{2}} \max _{\ell \in \mathbb{Z}}\left(\operatorname{Var}\left(\widehat{R}_{k^{2}}(\ell)\right)\right)+\max _{\ell \in \mathbb{Z}}\left(\mathbb{E}\left(\Delta_{\ell, k}^{*}\right)^{2}\right)\right) \leq \frac{c \cdot A}{k^{2}}
$$

for a constant $A>0$ depending on $\mathbb{E}\left|X_{0}\right|^{4}, \kappa_{4}$, and $\gamma$ only. Hence $b_{k} \leq 3(\gamma+A \cdot c) /\left(k^{2} t^{2}\right)$ is a summable series and, with $C_{2}>0$,

$$
B_{N} \leq \frac{C_{2}}{t^{2}} \cdot \sum_{k \geq \sqrt{N}} \frac{1}{k^{2}} .
$$

Then, (25), (26) and (27) imply $\sup _{n \geq N}\left\|J_{n}-J\right\|_{\mathcal{H}_{s}^{\prime}} \underset{N \rightarrow \infty}{\stackrel{\mathbb{P}}{\longrightarrow}} 0$ what is equivalent to $\left\|J_{n}-J\right\|_{\mathcal{H}_{s}^{\prime}} \underset{n \rightarrow \infty}{\stackrel{a . s .}{\longrightarrow}} 0$.

\subsection{Proofs of the section 2.3.1}

First let us recall the following classical lemma (see a proof in Rosenblatt (1985) [36], p. 58):

Lemma 4 If $X$ satisfies Assumption $M$ and $(\ell, k) \in \mathbb{Z}^{2}$ be arbitrary integers, then

$$
n \cdot \operatorname{Cov}\left(\widehat{R}_{n}(k), \widehat{R}_{n}(\ell)\right) \underset{n \rightarrow \infty}{\longrightarrow} \sigma_{k, \ell} .
$$


Proof of Lemma 目. Under condition (7), the projective criterion, introduced in Dedecker and Rio (2000), i.e. $\sum_{k \geq 0} \mathbb{E}\left|Y_{0, \ell_{i}} \mathbb{E}\left(Y_{k, \ell_{i}} \mid \sigma\left(Y_{j, \ell_{i}}, j \leq 0\right)\right)\right|<\infty$ for all $i \in\{1, \ldots, m\}$, holds. Therefore each $\widehat{R}_{n}\left(\ell_{i}\right)$ satisfies the central limit theorem. Now, by considering a a linear combination of $\left(Y_{j, \ell_{1}}, \ldots, Y_{j, \ell_{m}}\right)$, denoted $Z_{j}$, the projective criterion is also satisfied by $\left(Z_{j}\right)_{j \in \mathbb{Z}}$ yielding the multidimensional central limit theorem (8).

Proof of Theorem 园. We first prove the following lemma:

Lemma 5 (Tightness) If $X$ satisfies Assumption $M$ then the sequence of processes $\left(Z_{n}\right)_{n \in \mathbb{N}^{*}}$ is tight in $\mathcal{H}_{s}^{\prime}$.

Proof of Lemma 5. Following de Acosta (1970), for showing the tightness we only need to prove that the sequence is flatly concentrated, this means that

$$
\mathbb{E}\left(\left\|p_{L} Z_{n}\right\|_{\mathcal{H}_{s}^{\prime}}^{2}\right) \underset{L \rightarrow \infty}{\longrightarrow} 0
$$

where $p_{L}: \mathcal{H}_{s}^{\prime} \rightarrow F_{L}^{\prime}$ denotes the orthogonal projection on the closed linear subspace $F_{L}^{\prime} \subset \mathcal{H}_{s}^{\prime}$ generated by $\left(e_{\ell}\right)_{|\ell| \geq L}$ with $e_{\ell}(\lambda)=e^{i \ell \lambda}$ (also $F_{L} \subset \mathcal{H}_{s}$ denote the subspace generated by $\left(e_{\ell}\right)_{|\ell| \geq L}$ ). Then, for $L>0$,

$$
\left\|p_{L} Z_{n}\right\|_{\mathcal{H}_{s}^{\prime}}=\sup _{\|g\|_{\mathcal{H}_{s}}<1, g \in F_{L}}\left|Z_{n}(g)\right| .
$$

Thus, for $g=\sum_{|\ell| \geq L} g_{\ell} e_{\ell} \in F_{L}$ and $\|g\|_{\mathcal{H}_{s}}<1$, using again the decomposition (24), we obtain

$$
\left|Z_{n}(g)\right|^{2} \leq 3 n \cdot\left(\left|T_{1}(g)\right|^{2}+\left|T_{2}(g)\right|^{2}+\left|T_{3}(g)\right|^{2}\right) .
$$

First, from a Cauchy-Schwarz inequality, we have

$$
\begin{aligned}
n\left(\left|T_{1}(g)\right|^{2}+\left|T_{2}(g)\right|^{2}\right) \leq & n\left(\sum_{|\ell| \geq n \vee L}(1+|\ell|)^{-2 s} R(\ell)^{2} \sum_{|\ell| \geq n \vee L}(1+|\ell|)^{2 s} g_{\ell}^{2}\right. \\
& \left.\quad+\mathbb{I}_{\{L<n\}} \frac{1}{n^{2}} \sum_{L \leq|\ell|<n}|\ell|^{2}(1+|\ell|)^{-2 s} R(\ell)^{2} \sum_{L \leq|\ell|<n}(1+|\ell|)^{2 s} g_{\ell}^{2}\right) \\
\leq & n(1+|n \vee L|)^{-2 s} \sum_{|\ell| \geq n \vee L} R(\ell)^{2}+\mathbb{I}_{\{L<n\}} \sum_{L \leq|\ell|<n}|\ell|(1+|\ell|)^{-2 s} R(\ell)^{2} \\
\leq & \gamma\left(L(1+L)^{-2 s} \mathbb{1}_{\{L \geq n\}}+L(1+L)^{-2 s} \mathbb{I}_{\{L<n\}}\right) .
\end{aligned}
$$

Thus, we obtain

$$
\sup _{\|g\|_{\mathcal{H}_{s}}<1, g \in F_{L}} n\left(\left|T_{1}(g)\right|^{2}+\left|T_{2}(g)\right|^{2}\right) \underset{L \rightarrow \infty}{\longrightarrow} 0 .
$$

Also note that

$$
\begin{aligned}
\sqrt{n} T_{3}(g) & =\sqrt{n} \sum_{|\ell|<n}\left(\widehat{R}_{n}(\ell)-\mathbb{E} \widehat{R}_{n}(\ell)\right) g_{\ell} \\
n\left|T_{3}(g)\right|^{2} & \leq n \sum_{L \leq|\ell|<n}(1+|\ell|)^{-2 s}\left(\widehat{R}_{n}(\ell)-\mathbb{E} \widehat{R}_{n}(\ell)\right)^{2} \\
\mathbb{E}\left(\sup _{\|g\|_{\mathcal{H}_{s}}<1, g \in F_{L}} n\left|T_{3}(g)\right|^{2}\right) & \leq \sum_{L \leq|\ell|<n}(1+|\ell|)^{-2 s} \cdot \sup _{\ell}\left(n \operatorname{Var}\left(\widehat{R}_{n}(\ell)\right)\right) \leq \sum_{|\ell| \geq L}(1+|\ell|)^{-2 s}\left(\kappa_{4}+3 \gamma\right) .
\end{aligned}
$$

Since $\sum_{\ell \in \mathbb{Z}}(1+|\ell|)^{-2 s}<+\infty$, we deduce $\mathbb{E}\left(\sup _{\|g\|_{\mathcal{H}_{s}}<1,} n\left|T_{g \in F_{L}}(g)\right|^{2}\right) \underset{L \rightarrow \infty}{\longrightarrow} 0$. With (29) and (28), the proof ends.

Now the proof of Theorem 2 can be achieved. Indeed, the tightness allows to establish the functional central limit theorem. Moreover, $J_{n}(g)-\mathbb{E} J_{n}(g)=\sum_{\ell \in \mathbb{Z}} g_{\ell}\left(\widehat{R}_{n}(\ell)-\mathbb{E}\left(\widehat{R}_{n}(\ell)\right)\right.$, and therefore, from (画), the limiting covariance of the process $\left(Z_{n}\right)_{n \geq 1}$ is given by (5). More details of the finite dimensional convergence of the process $\left(Z_{n}\left(g_{1}\right), \ldots, Z_{n}\left(g_{k}\right)\right)$ can be found in Rosenblatt (1985, Corollary 2, p. 61). 
Proof of Corollary 日. Respectively from Rio's inequality (1994) and from X's stationarity, we have, for all $\ell, k \in \mathbb{N}$

$$
\begin{aligned}
\left\|Y_{0, \ell} \mathbb{E}\left(Y_{k+\ell, \ell} \mid \mathcal{B}_{\ell}\right)\right\|_{1} & \leq 2 \int_{0}^{\alpha\left(\sigma\left(Y_{k+\ell, \ell}\right), \mathcal{B}_{\ell}\right)} Q_{\mid Y_{0, \ell \mid}}(u) Q_{\left|Y_{k+\ell, \ell}\right|}(u) d u \\
& \leq 2 \int_{0}^{\alpha\left(\sigma\left(X_{\ell}, X_{k+\ell}\right), \mathcal{B}_{0}\right)} Q_{\left|Y_{0, \ell}\right|}^{2}(u) d u
\end{aligned}
$$

Therefore, for all $\ell, k \in \mathbb{N}$,

$$
\left\|Y_{0, \ell} \mathbb{E}\left(Y_{k+\ell, \ell} \mid \mathcal{B}_{\ell}\right)\right\|_{1} \leq 2 \int_{0}^{\alpha_{k}^{\prime}} Q_{\left|Y_{0, \ell}\right|}^{2}(u) d u
$$

Consequently, for all $\ell \in \mathbb{N}$

$$
\begin{aligned}
\sum_{k \geq 0}\left\|Y_{0, \ell} \mathbb{E}\left(Y_{k+\ell, \ell} \mid \mathcal{B}_{\ell}\right)\right\|_{1} & \leq 2 \int_{0}^{1}\left(\sum_{k \geq 0} \mathbb{I}_{u \leq \alpha_{k}^{\prime}}\right) Q_{\left|Y_{0, \ell}\right|}^{2}(u) d u \\
& \leq 2 \int_{0}^{1}\left(\alpha^{\prime}(u)\right)^{-1} Q_{\left|Y_{0, \ell}\right|}^{2}(u) d u .
\end{aligned}
$$

But Lemma 2.1 in Rio (2000) provides

$$
\begin{aligned}
\int_{0}^{1}\left(\alpha^{\prime}(u)\right)^{-1} Q_{\left|Y_{0, \ell}\right|}^{2}(u) d u & \leq \int_{0}^{1}\left(\alpha^{\prime}(u)\right)^{-1}\left(Q_{\left|X_{0}\right|}(u) Q_{\left|X_{\ell}\right|}(u)+Q_{|R(\ell)|}(u)\right)^{2} d u \\
& \leq \int_{0}^{1}\left(\alpha^{\prime}(u)\right)^{-1}\left(Q_{\left|X_{0}\right|}^{2}(u)+|R(\ell)|\right)^{2} d u,
\end{aligned}
$$

and therefore if $\int_{0}^{1}\left(\alpha^{\prime}(u)\right)^{-1} Q_{\left|X_{0}\right|}^{4}(u) d u<\infty$, then $\sum_{k \geq 0}\left\|Y_{0, \ell} \mathbb{E}\left(Y_{k, \ell} \mid \mathcal{B}_{\ell}\right)\right\|_{1}<\infty$ for all $\ell \in \mathbb{N}$.

Proof of Corollary 6 . We truncate the variables $X_{j}=f_{M}\left(X_{j}\right)+g_{M}\left(X_{j}\right)$ where, for $x \in \mathbb{R}$, we set $f_{M}(x)=$ $(x \wedge M) \vee(-M)$ (then $\left.f_{M} \in[-M, M]\right)$ and $g_{M}(x)=x-f_{M}(x)$ satisfies $\left|g_{M}(x)\right| \leq|x| \mathbb{I}_{|x| \geq M}$. Note that $\operatorname{Lip} f_{M}=1$ and $\left\|f_{M}\right\|_{\infty}=M$.

Then, $Y_{k, \ell}=\left(f_{M}\left(X_{k}\right) f_{M}\left(X_{k+\ell}\right)-R^{\prime}(\ell)\right)+U_{k, \ell, M}$ with:

- $R^{\prime}(\ell)=\mathbb{E} f_{M}\left(X_{k}\right) f_{M}\left(X_{k+\ell}\right)$

- $U_{k, \ell, M}=g_{M}\left(X_{k}\right) f_{M}\left(X_{k+\ell}\right)+f_{M}\left(X_{k}\right) g_{M}\left(X_{k+\ell}\right)+g_{M}\left(X_{k}\right) g_{M}\left(X_{k+\ell}\right)+R^{\prime}(\ell)-R(\ell)$.

Therefore, $\mathbb{E}\left(U_{k, \ell, M}\right)=0$ and, for $m$ such that $\left\|X_{0}\right\|_{m}<\infty$, we derive

$$
\begin{aligned}
\left\|U_{k, \ell, M}\right\|_{1} \leq M \mathbb{E}\left|X_{k}\right| \cdot \mathbb{I}_{\left|X_{k}\right|>M}+M \mathbb{E}\left|X_{k+\ell}\right| \cdot \mathbb{I}_{\left|X_{k+\ell}\right|>M} & +\mathbb{E}\left(\left(\left|X_{k}\right| \cdot \mathbb{I}_{\left|X_{k}\right|>M}\right)^{2}\right)+\left|\mathbb{E}\left(X_{0} X_{\ell}-f_{M}\left(X_{0}\right) f_{M}\left(X_{\ell}\right)\right)\right| \\
\leq & 2 M\left\|X_{0}\right\|_{m}\left(\mathbb{P}\left(X_{0}>M\right)\right)^{1-1 / m}+\left\|X_{0}\right\|_{m}^{2}\left(\mathbb{P}\left(X_{0}>M\right)\right)^{1-2 / m} \\
& +\mathbb{E}\left|f_{M}\left(X_{0}\right) g_{M}\left(X_{\ell}\right)\right|+\mathbb{E}\left|g_{M}\left(X_{0}\right) f_{M}\left(X_{\ell}\right)\right|+\mathbb{E}\left|g_{M}\left(X_{0}\right) g_{M}\left(X_{\ell}\right)\right| \\
\leq & 4 M\left\|X_{0}\right\|_{m}\left(\frac{\mathbb{E}\left|X_{0}\right|^{m}}{M^{m}}\right)^{1-1 / m}+2\left\|X_{0}\right\|_{m}^{2}\left(\frac{\mathbb{E}\left|X_{0}\right|^{m}}{M^{m}}\right)^{1-2 / m} \\
\leq & 6 \cdot M^{2-m} \cdot\left\|X_{0}\right\|_{m}^{m},
\end{aligned}
$$

from Hölder and Markov inequalities. By the same procedure, we also obtain

$$
\begin{aligned}
\left\|U_{k, \ell, M}\right\|_{2}^{2} & \leq 6\left(\mathbb{E} g_{M}^{2}\left(X_{k}\right) f_{M}^{2}\left(X_{k+\ell}\right)+\mathbb{E} f_{M}^{2}\left(X_{k}\right) g_{M}^{2}\left(X_{k+\ell}\right)+\mathbb{E} g_{M}^{2}\left(X_{k}\right) g_{M}^{2}\left(X_{k+\ell}\right)\right) \\
& \leq 18 \cdot M^{4-m} \cdot\left\|X_{0}\right\|_{m}^{m} .
\end{aligned}
$$


Let $h_{M}$ be the function such that $h_{M}(x, y)=f_{M}(x) f_{M}(y)-R^{\prime}(\ell)$ for all $(x, y) \in \mathbb{R}^{2}$. Note that $\left\|h_{M}\right\|_{\infty} \leq 2 M^{2}$ and $\operatorname{Lip} h_{M}=M$. Moreover, for all random variable $W$ in $\mathbb{L}^{1}(\Omega, \mathcal{A}, \mathbb{P})$,

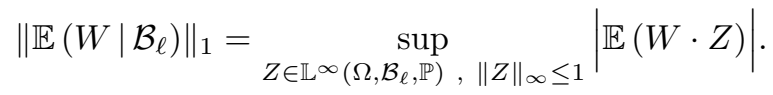

Therefore,

$$
\begin{aligned}
\left\|h_{M}\left(X_{0}, X_{\ell}\right) \cdot \mathbb{E}\left(h_{M}\left(X_{k}, X_{k+\ell}\right) \mid \mathcal{B}_{\ell}\right)\right\|_{1} & =\left\|\mathbb{E}\left(h_{M}\left(X_{0}, X_{\ell}\right) \cdot h_{M}\left(X_{k}, X_{k+\ell}\right) \mid \mathcal{B}_{\ell}\right)\right\|_{1} \\
& =\sup _{Z \in \mathbb{L}^{\infty}\left(\Omega, \mathcal{B}_{\ell}, \mathbb{P}\right),\|Z\|_{\infty} \leq 1}\left|\mathbb{E}\left(Z \cdot h_{M}\left(X_{0}, X_{\ell}\right) \cdot h_{M}\left(X_{k}, X_{k+\ell}\right)\right)\right| \\
& \leq \sup _{Z^{\prime} \in \mathbb{L}^{\infty}\left(\Omega, \mathcal{B}_{\ell}, \mathbb{P}\right),\left\|Z^{\prime}\right\|_{\infty} \leq 2 M^{2}}\left|\operatorname{Cov}\left(h_{M}\left(X_{k}, X_{k+\ell}\right), Z^{\prime}\right)\right| .
\end{aligned}
$$

Consequently, from the inequality (10) and the stationarity of $X$, for all $k \geq 0$,

$$
\left\|h_{M}\left(X_{0}, X_{\ell}\right) \cdot \mathbb{E}\left(h_{M}\left(X_{k}, X_{k+\ell}\right) \mid \mathcal{B}_{\ell}\right)\right\|_{1} \leq 4 \cdot M^{3} \cdot \theta_{k-|\ell|} .
$$

Thus,

$$
\begin{aligned}
\left\|Y_{0, \ell} \cdot \mathbb{E}\left(Y_{k, \ell} \mid \mathcal{B}_{\ell}\right)\right\|_{1} \leq & \left\|U_{0, \ell, M} \cdot \mathbb{E}\left(U_{k, \ell, M} \mid \mathcal{B}_{\ell}\right)\right\|_{1}+\left\|h_{M}\left(X_{0}, X_{\ell}\right) \mathbb{E}\left(U_{k, \ell, M} \mid \mathcal{B}_{\ell}\right)\right\|_{1} \\
& +\left\|U_{0, \ell, M} \cdot \mathbb{E}\left(h_{M}\left(X_{k}, X_{\ell+k}\right) \mid \mathcal{B}_{\ell}\right)\right\|_{1}+\left\|h_{M}\left(X_{0}, X_{\ell}\right) \cdot \mathbb{E}\left(h_{M}\left(X_{k}, X_{k+\ell}\right) \mid \mathcal{B}_{\ell}\right)\right\|_{1} \\
\leq & \left\|U_{k, \ell, M}\right\|_{2}^{2}+4 M^{2}\left\|U_{k, \ell, M}\right\|_{1}+\left\|h_{M}\left(X_{0}, X_{\ell}\right) \cdot \mathbb{E}\left(h_{M}\left(X_{k}, X_{k+\ell}\right) \mid \mathcal{B}_{\ell}\right)\right\|_{1} \\
\leq & 42 \cdot M^{4-m} \cdot\left\|X_{0}\right\|_{m}^{m}+4 \cdot M^{3} \cdot \theta_{k-|\ell|},
\end{aligned}
$$

from (30), (31) and (32). Set $M=\theta_{k-|\ell|}^{-1 /(m-1)}$, the previous calculations prove that, if there exists some $m>4$ such that

$$
\sum_{k=0}^{\infty} \theta_{k}^{1-3 /(m-1)}<\infty, \quad \text { and } \quad\left\|X_{0}\right\|_{m}<\infty
$$

then the Uniform CLT (9) holds.

\subsection{Proofs of Theorem 3 and Corollary 3}

Proof of Theorem 3. Set $g \in \mathcal{H}_{s}$ with $g(\lambda)=\sum_{\ell \in \mathbb{Z}} g_{\ell} e^{i \lambda \ell}$, let $k \in \mathbb{N}^{*}$ and define $g^{(k)}(\lambda)=\sum_{|\ell|<k} g_{\ell} e^{i \lambda \ell}$. One obtains for all $\mathcal{C}^{3}(\mathbb{R})$ function $\phi$ with bounded derivatives up to order 3

$$
\left|\mathbb{E}\left[\phi\left(\sqrt{n}\left(J_{n}(g)-J(g)\right)\right)-\phi(\sigma(g) \cdot N)\right]\right| \leq D_{1, n}^{(k)}+D_{2, n}^{(k)}+D_{3, n}^{(k)},
$$

with

$$
\begin{aligned}
D_{1, n}^{(k)} & =\left|\mathbb{E}\left[\phi\left(\sqrt{n}\left(J_{n}\left(g^{(k)}\right)-J\left(g^{(k)}\right)\right)\right)-\phi\left(\sigma\left(g^{(k)}\right) \cdot N\right)\right]\right| \\
D_{2, n}^{(k)} & =\left|\mathbb{E}\left[\phi\left(\sigma\left(g^{(k)}\right) \cdot N\right)-\phi(\sigma(g) \cdot N)\right]\right| \\
D_{3, n}^{(k)} & =\left|\mathbb{E}\left[\phi\left(\sqrt{n}\left(J_{n}\left(g^{(k)}\right)-J\left(g^{(k)}\right)\right)\right)-\phi\left(\sqrt{n}\left(J_{n}(g)-J(g)\right)\right)\right]\right| .
\end{aligned}
$$

$\operatorname{Term} D_{1, n}^{(k)}$ : For $i=1, \ldots, n$, set $x_{i}=\left(X_{i+\ell}\right)_{|\ell|<k}$ a stationary random vector in $\mathbb{R}^{2 k-1}$. The function

$$
h\left(x_{i}\right)=\sum_{|\ell|<k} g_{\ell}\left(X_{i} X_{i+\ell}-R(\ell)\right) \text { for } i=1, \ldots, n,
$$

satisfies the assumption $\mathrm{H}$ (namely $\mathbb{E} h\left(x_{0}\right)=0$ and there exists $a \geq 1$ and $A=A(d) \geq 1$ such as for all $u, v \in \mathbb{R}^{d},|h(u)| \leq A\left(|u|^{a} \vee 1\right)$ and $|h(u)-h(v)| \leq A\left(\left(|u|^{a-1}+|v|^{a-1}\right) \vee 1\right)|u-v|$, see in [2] $)$. Here $a=2$ and $A=A(2 k-1)=2 k-1$. Define also

$$
S_{n}^{(k)}=\frac{1}{\sqrt{n}} \sum_{i=1}^{n} h\left(x_{i}\right)=\sqrt{n}\left(J_{n}\left(g^{(k)}\right)-J\left(g^{(k)}\right)\right) .
$$


By applying Theorem 1 of Bardet et al. (2005) to this function $h$, one obtains with $C_{1}>0$ and $\lambda=\frac{\alpha(m-4)-2 m+1}{2(m+1+\alpha \cdot m}$,

$$
D_{1, n}^{(k)} \leq C_{1} \cdot k^{3} \cdot n^{-\lambda},
$$

$\operatorname{Term} D_{2, n}^{(k)}$ : From inequality $|a-b| \leq\left|a^{2}-b^{2}\right| / a$ for $a>0, b \geq 0$, and from the mean value theorem,

$$
D_{2, n}^{(k)} \leq \frac{\left\|\phi^{\prime}\right\|_{\infty}}{\sigma^{2}(g)}\left|\sigma^{2}(g)-\sigma^{2}\left(g^{(k)}\right)\right| .
$$

But, from the expression (5), we deduce

$$
\begin{aligned}
\left|\sigma^{2}(g)-\sigma^{2}\left(g^{(k)}\right)\right| \leq \mid \frac{1}{\pi} \int_{-\pi}^{\pi}\left(g^{2}(\lambda)-\left(g^{(k)}(\lambda)\right)^{2}\right) & f^{2}(\lambda) d \lambda \\
& +2 \pi \int_{-\pi}^{\pi} \int_{-\pi}^{\pi}\left(g(\lambda) g(\mu)-g^{(k)}(\lambda) g^{(k)}(\mu)\right) f_{4}(\lambda,-\mu, \mu) d \lambda d \mu \mid .
\end{aligned}
$$

With $g \in \mathcal{H}_{s}$, we have

$$
\left\|g-g^{(k)}\right\|_{\infty} \leq\left(\sum_{|\ell| \geq k}(1+|\ell|)^{-2 s}\right)^{1 / 2}\left(\sum_{|\ell| \geq k}(1+|\ell|)^{2 s} g_{\ell}^{2}\right)^{1 / 2} \leq\left(\sum_{|\ell| \geq k}(1+|\ell|)^{-2 s}\right)^{1 / 2}\|g\|_{\mathcal{H}_{s}} .
$$

Consequently, with also $\left\|g+g^{(k)}\right\|_{\infty} \leq 2\left(\sum_{\ell \in \mathbb{Z}}(1+|\ell|)^{-2 s}\right)^{1 / 2} \cdot\|g\|_{\mathcal{H}_{s}}$, there exists $C_{2}>0$ such that

$$
D_{2, n}^{(k)} \leq C_{2} \cdot k^{\frac{1}{2}-s}
$$

$\operatorname{Term} D_{3, n}^{(k)}$ : First, from a Taylor expansion,

$$
D_{3, n}^{(k)} \leq \frac{1}{2} \cdot\left\|\phi^{\prime \prime}\right\|_{\infty} \cdot n \cdot \mathbb{E}\left(J_{n}\left(g-g^{(k)}\right)-J\left(g-g^{(k)}\right)\right)^{2} .
$$

With the same decomposition as in the proof of Lemma 3 , one obtains

$$
\begin{aligned}
\mathbb{E}\left(J_{n}\left(g-g^{(k)}\right)-J\left(g-g^{(k)}\right)\right)^{2} \leq 3\left(\left(\sum_{|\ell| \geq n} R(\ell) g_{\ell}\right)^{2}\right. & +\left(\frac{1}{n} \sum_{k \leq|\ell|<n}|\ell| R(\ell) g_{\ell}\right)^{2}+ \\
& \left.+\left\|\sum_{k \leq|\ell|<n} g_{\ell}\left(\widehat{R}_{n}(\ell)-\mathbb{E}\left(\widehat{R}_{n}(\ell)\right)\right)\right\|_{2}^{2}\right) .
\end{aligned}
$$

First,

$$
\left(\sum_{|\ell| \geq n} R(\ell) g_{\ell}\right)^{2} \leq(1+n)^{-2 s} \cdot \sum_{|\ell| \geq n} R(\ell)^{2} \cdot \sum_{|\ell| \geq n}(1+|\ell|)^{2 s} g_{\ell}^{2} \leq \frac{1}{n} \cdot\|g\|_{\mathcal{H}_{s}}^{2} \cdot \sum_{|\ell| \geq n} R(\ell)^{2} .
$$

Using the weak dependence of $\left(X_{i}\right)_{i}$ and with the same method as in the proof of Lemma 2 in Bardet et al. (2005) adapted to the function $h(x)=x$ (therefore with $a=1$ ),

$$
|R(\ell)| \leq c \cdot \eta_{|\ell|}^{\frac{m-2}{m-1}} \leq c \cdot|\ell|^{-\alpha \frac{m-2}{m-1}}
$$

from the rate $\eta_{|\ell|}=\mathcal{O}\left(|\ell|^{-\alpha}\right)$ with $\alpha>3$. As a consequence,

$$
\sum_{|\ell| \geq n} R(\ell)^{2} \leq c \cdot n^{1-2 \alpha \frac{m-2}{m-1}} \text { and }\left(\sum_{|\ell| \geq n} R(\ell) g_{\ell}\right)^{2} \leq c \cdot n^{-2 \alpha \frac{m-2}{m-1}} .
$$

In the same way,

$$
\left(\frac{1}{n} \sum_{k \leq|\ell|<n}|\ell| R(\ell) g_{\ell}\right)^{2} \leq \frac{1}{n} \cdot\|g\|_{\mathcal{H}_{s}}^{2} \cdot \sum_{k \leq|\ell|<n} R(\ell)^{2} \leq \frac{c}{n} \cdot k^{1-2 \alpha \frac{m-2}{m-1}}
$$


Finally,

$$
\begin{aligned}
\left\|\sum_{k \leq|\ell|<n} g_{\ell}\left(\widehat{R}_{n}(\ell)-\mathbb{E}\left(\widehat{R}_{n}(\ell)\right)\right)\right\|_{2}^{2} & \leq\left(\sum_{k \leq|\ell|<n}\left|g_{\ell}\right|\left(\operatorname{Var}\left(\widehat{R}_{n}(\ell)\right)\right)^{1 / 2}\right)^{2} \\
& \leq \max _{\ell \in \mathbb{Z}}\left(\operatorname{Var}\left(\widehat{R}_{n}(\ell)\right)\right) \cdot\|g\|_{\mathcal{H}_{s}}^{2} \cdot \sum_{k \leq|\ell|<n}(1+|\ell|)^{-2 s} \\
& \leq \frac{1}{n} \cdot\left(\kappa_{4}+2 \gamma\right) \cdot\|g\|_{\mathcal{H}_{s}}^{2} \cdot \sum_{k \leq|\ell|<n}(1+|\ell|)^{-2 s} \text { from Lemma 2. }
\end{aligned}
$$

Summing up, there exists $C_{3}>0$ such that

$$
D_{3, n}^{(k)} \leq C_{3} \cdot\left(k^{1-2 \alpha \frac{m-2}{m-1}}+k^{1-2 s}\right) .
$$

Now, with (33), (34) and (35), we deduce by considering $t=\left(2 \alpha \frac{m-2}{m-1}-1\right) \wedge\left(s-\frac{1}{2}\right)$ and selecting $k$ such that $k^{t+3}=n^{\lambda}$, that there exists $C>0$ such that

$$
\left|\mathbb{E}\left[\phi\left(\sqrt{n}\left(J_{n}(g)-J(g)\right)\right)-\phi(\sigma(g) \cdot N)\right]\right| \leq C \cdot n^{-\frac{t}{t+3} \lambda}
$$

Proof of Corollary 3. Set $g(\lambda)=e^{i \ell \lambda}$ in Theorem 3. Since this function belongs to each space $\mathcal{H}_{s}$, it follows that the terms $D_{2, n}^{(k)}$ and $D_{3, n}^{(k)}$ both vanish and the result follows from the bound (33).

\section{Appendix: a useful lemma}

For a weakly dependent process, the following auxiliary lemma shows that a function of this process is also a weakly dependent process and moreover provides a relation between the two weak dependence sequences. For an example of the use of such a result, see the paragraph devoted to causal $\mathrm{ARCH}(\infty)$ time series.

Lemma 6 Let $\left(X_{i}\right)_{i \in \mathbb{Z}}$ be a $\mathbb{R}^{d}$-valued and $\mathbb{L}^{p}$-stationary time series with $p>1, h: \mathbb{R}^{d} \rightarrow \mathbb{R}$ be a function such that $|h(x)| \leq c\|x\|^{a}$ and $|h(x)-h(y)| \leq c\|x-y\|\left(\|x\|^{a-1}+\|y\|^{a-1}\right)$ for $(x, y) \in\left(\mathbb{R}^{d}\right)^{2}$, $\|\cdot\|$ a norm on $\mathbb{R}^{d}$, with $0<c$ and $1 \leq a<p$. Let $\left(Y_{i}\right)_{i \in \mathbb{Z}}$ be the stationary times series defined by $Y_{i}=h\left(X_{i}\right)$ for $i \in \mathbb{Z}$. Then

- If $\left(X_{i}\right)_{i \in \mathbb{Z}}$ is $\theta$-weakly dependent time series, then $\left(Y_{i}\right)_{i \in \mathbb{Z}}$ is a stationary $\theta^{Y}$-weakly dependent time series, such that $\forall r \in \mathbb{N}, \theta_{r}^{Y}=C \cdot \theta_{r}^{\frac{p-a}{p-1}}$ with a constant $C>0$;

- If $\left(X_{i}\right)_{i \in \mathbb{Z}}$ is $\eta$-weakly dependent time series, then $\left(Y_{i}\right)_{i \in \mathbb{Z}}$ is a $\eta^{Y}$-weakly dependent time series, such that $\forall r \in \mathbb{N}, \eta_{r}^{Y}=C \cdot \eta_{r}^{\frac{p-a}{p-1}}$ with a constant $C>0$.

Proof. We first assume that $d=1$. Let $f: \mathbb{R}^{u} \rightarrow \mathbb{R}$ and $g: \mathbb{R}^{v} \rightarrow \mathbb{R}$ two real functions such that Lip $f<\infty$, $\|f\|_{\infty} \leq 1, \operatorname{Lip} g<\infty,\|g\|_{\infty} \leq 1$. Denote $x^{(M)}=(x \wedge M) \vee(-M)$ for $x \in \mathbb{R}$. For simplicity we first assume that $v=2$. Let $i_{1}, \ldots, i_{u}, j_{1}, \ldots, j_{v} \in \mathbb{Z}^{u+v}$ such that $i_{1}, \ldots, i_{u} \geq r$ and $j_{1}, \ldots, j_{v} \leq 0$ and denote $x_{\mathbf{i}}=\left(X_{i_{1}}, \ldots, X_{i_{u}}\right)$ and $x_{\mathbf{j}}=\left(X_{j_{1}}, \ldots, X_{j_{v}}\right)$. We then define functions $F: \mathbb{R}^{u} \rightarrow \mathbb{R}, F^{(M)}: \mathbb{R}^{u} \rightarrow \mathbb{R}$ and $G: \mathbb{R}^{v} \rightarrow \mathbb{R}$, $G^{(M)}: \mathbb{R}^{v} \rightarrow \mathbb{R}$ through the relations, $F\left(x_{\mathbf{i}}\right)=f\left(h\left(X_{i_{1}}\right), \ldots, h\left(X_{i_{u}}\right)\right), F^{(M)}\left(x_{\mathbf{i}}\right)=f\left(h\left(X_{i_{1}}^{(M)}\right), \ldots, h\left(X_{i_{u}}^{(M)}\right)\right)$ and $G\left(x_{\mathbf{j}}\right)=g\left(h\left(X_{j_{1}}\right), \ldots, h\left(X_{j_{v}}\right)\right), G^{(M)}\left(x_{\mathbf{j}}\right)=g\left(h\left(X_{j_{1}}^{(M)}\right), \ldots, h\left(X_{j_{v}}^{(M)}\right)\right)$. Then

$$
\begin{aligned}
\left|\operatorname{Cov}\left(F\left(x_{\mathbf{i}}\right), G\left(x_{\mathbf{j}}\right)\right)\right| & \leq\left|\operatorname{Cov}\left(F\left(x_{\mathbf{i}}\right), G\left(x_{\mathbf{j}}\right)-G^{(M)}\left(x_{\mathbf{j}}\right)\right)\right|+\left|\operatorname{Cov}\left(F\left(x_{\mathbf{i}}\right), G^{(M)}\left(x_{\mathbf{j}}\right)\right)\right| \\
& \left.\leq 2 \mathbb{E} \mid G\left(x_{\mathbf{j}}\right)-G^{(M)}\left(x_{\mathbf{j}}\right)\right)|+2 \mathbb{E}| F\left(x_{\mathbf{i}}\right)-F^{(M)}\left(x_{\mathbf{i}}\right)|+| \operatorname{Cov}\left(F^{(M)}\left(x_{\mathbf{i}}\right), G^{(M)}\left(x_{\mathbf{j}}\right)\right) \mid .
\end{aligned}
$$

The last relation comes from $\|f\|_{\infty} \leq 1$. But we also have

$$
\begin{aligned}
\left.\mathbb{E} \mid G\left(x_{\mathbf{j}}\right)-G^{(M)}\left(x_{\mathbf{j}}\right)\right) \mid & \leq v \cdot \operatorname{Lip} g \cdot \mathbb{E}\left|h\left(X_{0}\right)-h\left(X_{0}^{(M)}\right)\right| \\
& \leq 2 c \cdot v \cdot \operatorname{Lip} g \cdot \mathbb{E}\left(\left|X_{0}\right|^{a} \cdot \mathbb{1}_{\left|X_{0}\right|>M}\right) \quad \text { (from the assumptions on } h \text { ), } \\
& \leq 2 c \cdot v \cdot \operatorname{Lip} g \cdot\left\|X_{0}\right\|_{p} \cdot M^{a-p} \quad \text { (from Markov inequality). }
\end{aligned}
$$

The same thing holds for $F$. Moreover, the functions $F^{(M)}$ and $G^{(M)}$ satisfy $\operatorname{Lip} F^{(M)}=\operatorname{Lip} F^{(M)}=c \cdot M^{a-1}$, with $c>0$, and $\left\|F^{(M)}\right\|_{\infty} \leq 1,\left\|G^{(M)}\right\|_{\infty} \leq 1$. Thus, from the definition of the weak dependence of $X$

$$
\begin{aligned}
\left|\operatorname{Cov}\left(F^{(M)}\left(x_{\mathbf{i}}\right), G^{(M)}\left(x_{\mathbf{j}}\right)\right)\right| & \leq C v \operatorname{Lip} f M^{a-1} \theta_{r}, \text { with } u=2, \text { under condition } \theta \\
& \leq C(v \operatorname{Lip} f+u \operatorname{Lip} g) M^{a-1} \eta_{r}, \quad \text { under condition } \eta
\end{aligned}
$$


Finally, we obtain respectively

$$
\begin{aligned}
\left|\operatorname{Cov}\left(F\left(x_{\mathbf{i}}\right), G\left(x_{\mathbf{j}}\right)\right)\right| & \leq C v \operatorname{Lip} f\left(M^{a-1} \theta_{r}+M^{a-p}\right) \\
& \leq C(v \operatorname{Lip} f+u \operatorname{Lip} g)\left(M^{a-1} \eta_{r}+M^{a-p}\right) .
\end{aligned}
$$

By the optimal choice of $M=\theta_{r}^{1 /(1-p)}$, we get respectively

$$
\begin{aligned}
\left|\operatorname{Cov}\left(f\left(Y_{i_{1}}, \ldots, Y_{i_{u}}\right), g\left(Y_{i_{1}}, \ldots, Y_{i_{v}}\right)\right)\right| & \leq C v \operatorname{Lip} f \theta_{r}^{\frac{p-a}{p-1}} \\
& \leq C(v \operatorname{Lip} f+u \operatorname{Lip} g) \eta_{r}^{\frac{p-a}{p-1}} .
\end{aligned}
$$

Changes for the vector valued case are simple, here truncations should only be considered componentwize and yield the same result up to a constant.

\section{References}

[1] Ango'nze, P., Doukhan, P. (2004) Weak dependence: models and applications to econometrics. Econometric Theory 20, 995-1045.

[2] Bardet, J.-M., Doukhan, P., León, J.R. (2005) A functional limit theorem for non causal weak dependent processes and its applications. Preprint of Samos, Université Paris 1, available with ftp://samos.univ-paris1.fr/pub/SAMOS/preprints/samos232.pdf.

[3] Bollerslev, T. (1986) Generalized autoregressive conditional heteroskedasticity. J. Econometrics 31, $307-327$.

[4] Brockwell, P.J., Davis, R.A. (1991) Time series: theory and methods. Second edition. Springer Series in Statistics. Springer-Verlag, New York.

[5] Dahlhaus, R., Polonik, W. (2002) Empirical spectral processes and nonparametric maximum likelihood estimation for time series., in Empirical Process Techniques for Dependent Data, Dehling, Mikosch and Sorensen editors, Birkhauser, 275-298.

[6] Dahlhaus, R. (1988) Empirical spectral processes and their applications to times series analysis. Stoch. Proc. Appl. 30, 69-83.

[7] Dalla, V., Giraitis, L. and Hidalgo, J. (2006) Consistent estimation of the memory parameter for nonlinear time series. J. Time Ser. Anal. 27, 211-251.

[8] De Acosta, A. (1970) Existence and convergence of probability measures in Banach spaces. Trans. Am. Math. Soc. 152, 273-298.

[9] Dedecker, J., Doukhan, P. (2003) A new covariance inequality and applications. Stoch. Proc. Appl. 106-1, 63-80.

[10] Dedecker, J., Louhichi, S. (2002) Maximal inequalities and empirical central limit theorems, in Empirical Process Techniques for Dependent Data, Dehling, Mikosch and Sorensen editors, Birkhaüser, 137-159.

[11] Dedecker, J., Rio, E. (2000) On the functional central limit theorem for stationary processes. Ann. Inst. H. Poincaré, Probab. Statist. 36, 1-34.

[12] Dehling, H., Mikosch T., Sorensen, M. (2002) Empirical Processes Techniques for Dependent Data (editors), Birkhaäuser.

[13] Doukhan, P. (2003) Models inequalities and limit theorems for stationary sequences, in Theory and applications of long range dependence (Doukhan et alii ed.) Birkhäuser, 43-101.

[14] Doukhan, P., Lang, G. (2002) Rates in the empirical central limit theorem for stationary weakly dependent random fields. Stat. Inference Stoch. Process. 5, 199-228.

[15] Doukhan, P., León, J. R. (1989) Cumulants for mixing sequences and applications to empirical spectral density. Probab. Math. Stat. 10.1, 11-26.

[16] Doukhan, P., Louhichi, S. (1999) A new weak dependence condition and applications to moment inequalities. Stoch. Proc. Appl. 84, 313-342. 
[17] Doukhan, P., Madre, H., Rosenbaum, M. (2006) ARCH-type bilinear weakly dependent models. To appear in Statistics.

[18] Doukhan, P., Teyssière, G., Winant, P. (2006) Vector valued ARCH( $\infty$ ) processes. To appear in Lecture Note in Statistics, Special issue on time series, Bertail, P. , Doukhan, P. and Soulier, P. Editors.

[19] Doukhan, P., Wintenberger, O. (2006) Invariance principle for new weakly dependent stationary models under sharp moment assumptions. To appear in Probab. Math. Statist.

[20] Engle, R. F. (1982) Autoregressive conditional heteroscedasticity with estimates of the variance of United Kingdom inflation. Econometrica 50, 987-1007.

[21] Fox, R., Taqqu, M. S. (1986). Large-sample properties of parameter estimates for strongly dependent Gaussian time series. Ann. Statist. 14, 517-532.

[22] Giraitis, L., Leipus, R., Surgailis, D. (2005). Recent advances in ARCH modelling. In Long-Memory in Economics. G. Teyssière and A. Kirman editors, Springer Verlag (Forthcoming).

[23] Giraitis, L., Surgailis, D. (1990). A central limit theorem for quadratic forms in strongly dependent linear variables and its application to asymptotical normality of Whittle's estimate. Probab. Th. Rel. Fields, 86, 87-104.

[24] Giraitis, L., Surgailis, D. (2002). ARCH-type bilinear models with double long memory. Stoch. Proc. Appl., 100, 275-300.

[25] Giraitis, L., Robinson, P.M. (2001). Whittle estimation of ARCH models. Econometric Theory 17, 608-631.

[26] Hall, P., Heyde, C. (1980) Martingale limit theory and its application. Academic Press, New York.

[27] Hannan, E. J. (1973) The asymptotic theory of linear time series models. J. Appl. Probab. 10, $130-145$.

[28] Horvath, L., Shao, Q.-M. (1999) Limit theorems for quadratic forms with applications to Whittle's estimate. Ann. Appl. Probab., 9, 146-187.

[29] Mikosch, T., Norvaisa, R. (1997) Uniform convergence of the empirical spectral distribution function. Stoch. Proc. Applic. 70, 85-114.

[30] Mikosch, T., Straumann, D. (2002) Whittle estimation in heavy-tailed GARCH(1,1) model. Stoch. Proc. Applic. 100, 187-222.

[31] Oliveira, P.E., Suquet, C. (1998) Weak convergence in $L_{p}[0,1]$ of the uniform empirical process under dependence. Statist. Probab. Letters 39-4, 363-370.

[32] Rio, E. (1994) Inégalités de moments pour les suites stationnaires et fortement mélangeantes. C.R. Acad. Sci. Paris 318, 355-360.

[33] Rio, E. (2000) Théorie asymptotique des processus aléatoires faiblement mélangeants. Collection Mathématiques \& Applications 31. Springer. Berlin.

[34] Robinson, P. M. (1991) Testing for strong serial correlation and dynamic conditional heteroskedasticity in multiple regression. J. Econometrics 47, 67-84.

[35] Robinson, P. M. (1995) Gaussian semiparametric estimation of long range dependence. Ann. Statist. 23, 1630-1661.

[36] Rosenblatt, M. (1985) Stationary processes and random fields. Birkhäuser, Boston.

[37] Rosenblatt, M. (2000) Gaussian and non-Gaussian linear time series and random fields. Springer Series in Statistics. Springer-Verlag, New York.

[38] Saulis, L., Statulevicius, V.A. (1991) Limit theorems for large deviation. Birkhäuser.

[39] Soulier, P. (2002) Estimation processes techniques for the spectral estimation of fractional processes, in Empirical Process Techniques for Dependent Data, Dehling, Mikosch and Sorensen editors, Birkhauser, 299-323.

[40] Straumann, D. (2005) Estimation in Conditionally Heteroscedastic Times Series Models. Lectures Notes in Statistics 181, Springer, New York.

[41] Zaffaroni, P. (2003) Gaussian inference on certain long-range dependent volatility models. J. Econometrics, $115,199-258$. 